\title{
Antimalarial Activity of Plant Metabolites
}

\author{
Wen-Hui Pan ${ }^{1}$, Xin-Ya Xu ${ }^{1,2}$, Ni Shi ${ }^{1}$, Siu Wai Tsang ${ }^{1}$ and Hong-Jie Zhang ${ }^{1, *}$ (i) \\ 1 School of Chinese Medicine, Hong Kong Baptist University, 7 Baptist University Road, \\ Kowloon Tong, Kowloon, Hong Kong SAR, China; 13480448@life.hkbu.edu.hk (W.-H.P.); \\ xuxinya@scsio.ac.cn (X.-Y.X.); 14252252@life.hkbu.edu.hk (N.S.); tsang@hkbu.edu.hk (S.W.T.); \\ 2 CAS Key Laboratory of Tropical Marine Bio-resources and Ecology, Guangdong Provincial Key Laboratory \\ of Applied Marine Biology, South China Sea Institute of Oceanology, Chinese Academy of Science, \\ Guangzhou 510070, China \\ * Correspondence: zhanghj@hkbu.edu.hk; Tel.: +852-3411-2956; Fax: +852-3411-2461
}

Received: 13 April 2018; Accepted: 2 May 2018; Published: 6 May 2018

\begin{abstract}
Malaria, as a major global health problem, continues to affect a large number of people each year, especially those in developing countries. Effective drug discovery is still one of the main efforts to control malaria. As natural products are still considered as a key source for discovery and development of therapeutic agents, we have evaluated more than 2000 plant extracts against Plasmodium falciparum. As a result, we discovered dozens of plant leads that displayed antimalarial activity. Our phytochemical study of some of these plant extracts led to the identification of several potent antimalarial compounds. The prior comprehensive review article entitled "Antimalarial activity of plant metabolites" by Schwikkard and Van Heerden (2002) reported structures of plant-derived compounds with antiplasmodial activity and covered literature up to the year 2000. As a continuation of this effort, the present review covers the antimalarial compounds isolated from plants, including marine plants, reported in the literature from 2001 to the end of 2017. During the span of the last 17 years, 175 antiplasmodial compounds were discovered from plants. These active compounds are organized in our review article according to their plant families. In addition, we also include ethnobotanical information of the antimalarial plants discussed.
\end{abstract}

Keywords: anti-malaria activity; plants; natural products; ethnopharmacology; Plasmodium parasites

\section{Introduction}

Malaria is still considered as a major global health problem, affecting a large population of the world. According to World Health Organization (WHO), there were about 216 million malaria cases globally and 445,000 deaths in 2016. Most of the cases and the deaths occurred in the WHO African region and affected primarily children and pregnant women [1].

P. falciparum, P. vivax, P. ovale, P. malariae and P. knowlesi are the five Plasmodium species that cause malaia disease in humans. P. falciparum is the deadliest strain that causes malaria and this form of parasite predominates in Africa [2,3]. Humans get infected with malaria parasites through the bites of female anopheline mosquitoes [4]. The Plasmodium parasites travel through blood and become mature and reproduce in the liver, leading to malaria disease. The common symptoms of malaria are fever and headache, and in severe cases, malaria causes death [5].

Currently, there is no commercially available malaria vaccine, though efforts to develop vaccines are still ongoing. The most promising vaccine candidate is RTS, S/AS01, which is in clinical trials for treatment of malaria caused by P. falciparum [1]. Several medications are available to prevent malaria for travellers in malaria-endemic countries, and a number of drugs are available for treatment of those who have the disease [6]. 
In 1820, French scientists Pelletier and Caventou discovered quinine (I) as the first antimalarial drug, which was originally isolated from the barks of Cinchona species (Rubiaceae) (Figure 1). Cinchona plants are used as folk medicines in South America by Peruvian Indians, and they were introduced to Europe in the 1700s [7]. Quinine is commercially obtained by solvent extraction from wild-growing Cinchona species in South America, or the plants cultivated in Indonesia [8].

Chloroquine (II) and its derivative 4-aminoquinoline were developed in the 1940s. They are widely used as antimalarial drugs, even today. The effectiveness of the drugs, however, has declined rapidly since the 1960s, which was due to the development of drug resistance by P. falciparum strains, leading to a significant malaria-associated death rate [9]. Mefloquine (III), is a 4-quinolinemethanol derivative obtained via total synthesis. It was introduced as a new antimalarial drug in 1985 . The drug can be used to treat mild or moderate malaria but should not be used to treat severe malaria [10].

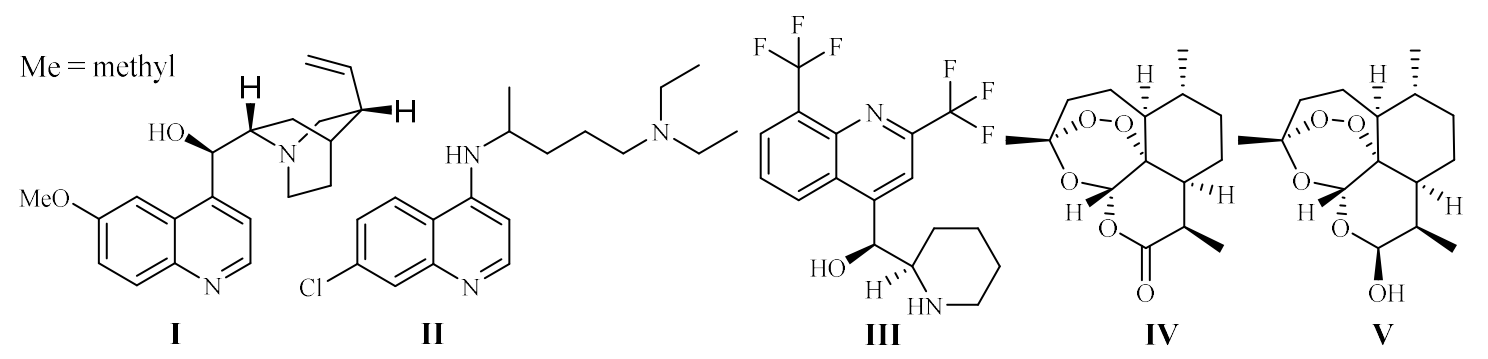

Figure 1. Antimalarial drugs developed from plants.

The current antimalarial drug of choice is artemisinin (Qinghaosu, IV), which was originally obtained from the leaves of Qinghao [Artemisia annua L. (Asteraceae)] in the 1970s. The compound is clinically effective against chloroquine-resistant malaria strains [11]. The plant Qinghao has been used as a traditional medicine in China for the treatment of fever of malaria origin for about 2000 years [12]. A large number of artemisinin analogs have also been synthesized. The best known among these derivatives are artemether, arteether (artemotil), artesunate and artenimol ( $\beta$-dihydroartemisinin, DHA) [13]. Artemisinin and its semi-synthetic derivatives have shown better efficacy than quinine for both children and adults patients [14].

Although the anti-parasitic mechanism of action of artemisinin is still in question [15], the endoperoxide bridge is regarded as the key functional group responsible for eliciting free radical-mediated parasite killing mechanisms. According to one school of thought, Plasmodium parasites live and reproduce in the host by ingesting red blood cell hemoglobin. This results in an accumulation of heme $\mathrm{Fe}^{2+}$ in the parasite. $\mathrm{Fe}^{2+}$ firstly interacts and cleaves the peroxide bridge of artemisinin to form highly reactive free radicals, which in turn cause a series of parasite molecular events and eventually kill the parasites [16]. The most used artemisinin derivative today is the prodrug, dihydroartemisinin $(\mathbf{V})$, which is metabolized into the pharmacologically active artimisinin (IV) in the body [17]. Artesunate was investigated as a potential inhibitor of the essential P. falciparum exported protein 1 (EXP1), a membrane glutathione $S$-transferase [18].

Clinically, it is unwise to use artemisinin as the lone therapy due to the potential risk of the parasites to develop resistance to this drug. Indeed, artemisinin drug resistance has been already detected in some Southern Asian countries: Lao People's Democratic Republic, Cambodia, Thailand, Myanmar and Viet Nam [1]. This risk has led to the withdrawal of artemisinin monotherapy from clinical applications.

At present, the use of artemisinins in combination with other drugs, known as artemisinin-based combination therapy (ACT), is the most effective to treat malarial disease caused by P. falciparum infection. Five currently available ACTs are artemether in combination with lumefantrine, and four other forms based on artesunate in combination with amodiaquine (two formulations), mefloquine and sulfadoxine+pyrimethamine [1]. Unfortunately, resistance has already been detected to both 
artemisinin and artesunate components of the multiple ACTs, as well as the non-artemisinin-based combination comprising atovaquone and proguanil. The current availbale antimalarial drugs are listed in Table $1[1,19,20]$.

Table 1. Available antimalarial drugs.

\begin{tabular}{|c|c|c|c|}
\hline Chemical Class & Generic Names & Chemical Class & Generic Names \\
\hline 4-Aminoquinolines & $\begin{array}{l}\text { chloroquine } \\
\text { amodiaquine } \\
\text { piperaquine }\end{array}$ & Antibiotics & $\begin{array}{l}\text { azythromycin } \\
\text { clindamycin } \\
\text { doxycycline }\end{array}$ \\
\hline 8-Aminoquinoline & $\begin{array}{l}\text { primaquine } \\
\text { bulaquine }\end{array}$ & \multirow{3}{*}{$\begin{array}{c}\text { Artemisinin-based combination } \\
\text { therapy (ACT) }\end{array}$} & $\begin{array}{l}\text { artemether-lumefantrine } \\
\text { artesunate }\end{array}$ \\
\hline Arylamino-alcohols & $\begin{array}{l}\text { quinine } \\
\text { quinidine } \\
\text { mefloquine } \\
\text { halofantrine } \\
\text { lumefantrine }\end{array}$ & & $\begin{array}{c}\text { artesunate/sulfadoxine/pyrimethamine } \\
\text { artesunate/sulfadoxine-pyrimethamine/primaquine } \\
\text { artesunate/amodiaquine } \\
\text { artesunate/mefloquine } \\
\text { artesunate/pyronaridine }\end{array}$ \\
\hline Biguanides & $\begin{array}{c}\text { proguanil } \\
\text { chlorproguanil }\end{array}$ & & $\begin{array}{l}\text { chloroquine/primaquine } \\
\text { dihydroartemisinin/piperaquine }\end{array}$ \\
\hline Glycosylamines & $\begin{array}{l}\text { pyrimethamine } \\
\text { proguanil } \\
\text { cycloguanil } \\
\text { chlorproguanil } \\
\text { chlorcycloguanil }\end{array}$ & \multirow[t]{2}{*}{$\begin{array}{l}\text { Antibiotics-antimalarial drug } \\
\text { combination }\end{array}$} & $\begin{array}{l}\text { doxycyclin/quinine } \\
\text { doxycycline/artesunate } \\
\text { doxycyclin/mefloquine } \\
\text { clindamycin/quinine } \\
\text { clindamycin/artesunate }\end{array}$ \\
\hline Naphthoquinone & atovaquone & & clindamycin/mefloquine \\
\hline $\begin{array}{l}\text { Sesquiterpene } \\
\text { lactones }\end{array}$ & $\begin{array}{l}\text { artemisinin } \\
\text { arteether } \\
\text { artemether } \\
\text { artesunate } \\
\text { dihydroartemisinin }\end{array}$ & Other combination therapy & $\begin{array}{l}\text { sulfadoxine/pyrimethamine } \\
\text { bulaquine/chloroquine } \\
\text { dapsone/chlorproguanil } \\
\text { atovaquone/proguanil }\end{array}$ \\
\hline Sulfonamides/Sulfones & $\begin{array}{l}\text { sulfadoxine } \\
\text { sulfalene } \\
\text { dapsone }\end{array}$ & & \\
\hline
\end{tabular}

In the search for drug candidates, the initial step is the employment of appropriate bioassays to evaluate the antiplasmodial activity of a candidate. Several strains of $P$. falciparum have been used for this purpose in the past. The strains of $P$. falciparum that are sensitive and resistant to chloroquine are frequently used for antimalarial drug discovery programs. D6, D10, 3D7, TM4 and PoW are chloroquine-sensitive strains, whereas, W2, FCR-3, FcB1 and Dd2 represent chloroquine-resistant strains, and $\mathrm{K} 1$ is a multidrug resistant strain.

The need to discover effective and non-drug resistant antimalarial drugs is urgent as Plasmodium strains have already developed resistance to all of today's available drugs including artemisinin. In that regard, it should be noted that natural products have proven to be a valuable source for the discovery of novel antimalarial therapeutic agents since the discovery of the first antimalarial drug in 1800s [20]. We, thus, pursued this approach in the search for new antimalarial potential drug leads.

In our antimalarial drug discovery program, we have evaluated more than 2000 plant extracts against D6 and W2 strains of P. falciparum. Dozens of these plants displayed antimalarial activity. Several of these plant leads were investigated further to uncover their antimalarial constituents. Phytochemical separation of these plant leads guided by bioassays led to the identification of ten new and 13 known active compounds [21]. Some of these compounds demonstrated potent antimalarial activity [22-29]. For example, polysyphorin (1) and rhaphidecurperoxin (2), isolated from Rhaphidophora decursiva (Araceae), showed antimalarial activities of 1.5 and $1.4 \mu \mathrm{M}$ against the W2 clones of P. falciparum, respectively (Figure 2) [22]. Two trichothecenes, roridin E (3) from R. decursiva (Araceae) and verrucarin L acetate (4) from Ficus fistulosa (Moraceae), were found to potently inhibit the parasite growth with $\mathrm{IC}_{50}$ values in the sub-nano molar range [24]. 


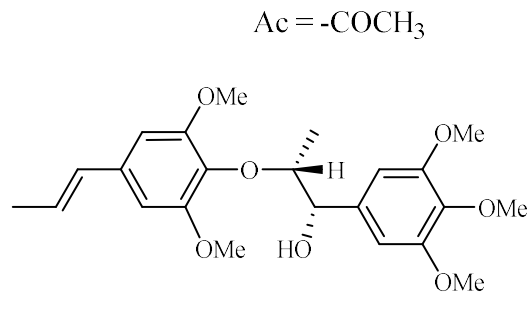

1

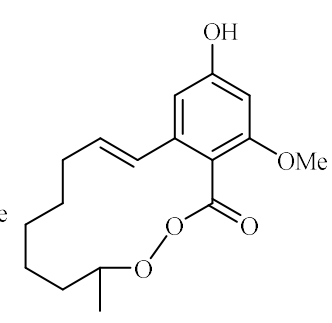

2
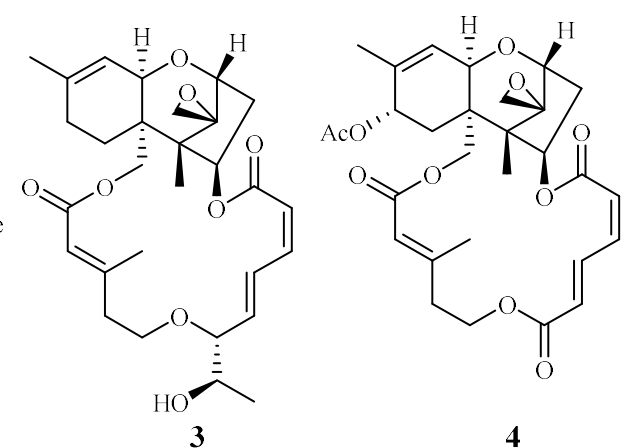

4

Figure 2. Compounds from R. decursiva and F. fitulosa.

De-replication to avoid duplication of previous efforts is an essential step in drug discovery protocols. To that end, we conducted a thorough review of the published literature on natural products possessing antimalarial activity. Previously, a literature review by Schwikkard and Van Heerden [30], covered plant-derived antiplasmodial active natural compounds up to the year 2000. The compounds were organized according to the origins of their corresponding plant families. The current review seeks to supplement the review of Schwikkard and Van Heerden. Compounds with antimalarial activity will also be organized according to their plant family of origin (Table 2). Literature published between 2001 and 2017 have been covered. In addition, we also included the ethnobotanic information of plants that have been used as folk medicines for the treatment of malarial disease (Table 3). 
Table 2. Antiplasmodial activities and toxicities of compounds isolated from terrestrial plants.

\begin{tabular}{|c|c|c|c|c|c|c|}
\hline Family & Species & Extract Solvent & Compound & $\begin{array}{c}\text { Antiplasmodial } \mathrm{IC}_{50}(\mu \mathrm{M})^{\mathrm{a}} \\
(\text { P. } \text { falciparum })^{2}\end{array}$ & $\begin{array}{c}\text { Cytotoxicity, } \mathrm{ED}_{50}(\mu \mathrm{M})^{\mathrm{b}} \\
\text { (Cell Line) }^{\mathrm{b}}\end{array}$ & References \\
\hline \multirow{6}{*}{ Annonaceae } & \multirow{3}{*}{ Friesodielsia discolor } & \multirow{3}{*}{ EtOAc } & 30-formyl-20,40-dihydroxy-60-methoxychalcone (5) & $9.2(\mathrm{~K} 1)$ & 21.8 (KB); 13.9 (MCF-7) & [31] \\
\hline & & & 8-formyl-7-hydroxy-5-methoxyflavanone (6) & $9.3(\mathrm{~K} 1)$ & 41.9 (KB); 34.5 (MCF-7) & \\
\hline & & & tectochrysin $(7)$ & $7.8(\mathrm{~K} 1)$ & 59.1 (KB); 16.8 (MCF-7) & \\
\hline & Mitrephora diversifolia & $\mathrm{CH}_{2} \mathrm{Cl}_{2} / \mathrm{MeOH}$ & 5-hydroxy-6-methoxyonychine (8) & 9.9 (3D7); $11.4(\mathrm{Dd} 2)$ & 120.0 (HEK293) & [32] \\
\hline & \multirow{2}{*}{ Miliusa cuneata } & \multirow{2}{*}{ Acetone } & miliusacunines A (9) & 19.3 (TM4) & - & [33] \\
\hline & & & miliusacunines B (10) & $10.8(\mathrm{~K} 1)$ & - & \\
\hline \multirow{8}{*}{ Araceae } & \multirow{8}{*}{ Rhaphidophora decursiva } & \multirow{8}{*}{$\mathrm{MeOH}$} & polysyphorin (1) & 1.7 (D6); $1.5(\mathrm{~W} 2)$ & $8.3(\mathrm{~KB})$ & {$[22,23]$} \\
\hline & & & rhaphidecurperoxin (2) & $1.8(\mathrm{D} 6) ; 1.4(\mathrm{~W} 2)$ & $13.1(\mathrm{~KB})$ & \\
\hline & & & rhaphidecursinol A (11) & 7.2 (D6); $4.2(\mathrm{~W} 2)$ & $28.7(\mathrm{~KB})$ & \\
\hline & & & rhaphidecursinol B (12) & 12.9 (D6); 11.2 (W2) & 23.9 (KB) & \\
\hline & & & grandisin (13) & $3.5(\mathrm{D} 6) ; 3.4(\mathrm{~W} 2)$ & $32.4(\mathrm{~KB})$ & \\
\hline & & & epigrandisin (14) & $>23(\mathrm{D} 6) ; 7.7$ (W2) & $37.0(\mathrm{~KB})$ & \\
\hline & & & decursivine (15) & 11.2 (D6); 12.6 (W2) & - & {$[22,23]$} \\
\hline & & & Roridin E (3) & 0.0004 (D6); $0.001(\mathrm{~W} 2)$ & $0.0004(\mathrm{~KB})$ & [24] \\
\hline Asclepiadaceae & Gongronema napalense & EtOH & gongroneside A (16) & $1.6(\mathrm{D} 6) ; 1.4(\mathrm{~W} 2)$ & $>13.7(\mathrm{~KB})$ & [25] \\
\hline \multirow{7}{*}{ Asteraceae } & \multirow{2}{*}{ Achillea millefolium } & \multirow{2}{*}{$\mathrm{MeOH}$} & apigenin 7-O-glucoside (17) & 25.3 (D10); 15.3 (W2) & - & [34] \\
\hline & & & luteolin 7-O-glucoside (18) & 61.1 (D10); 62.5 (W2) & - & \\
\hline & Carpesium divaricatum & $\mathrm{MeOH}$ & 2-isopropenyl-6-acetyl-8-methoxy-1,3-benzodioxin-4-one (19) & $2.3(\mathrm{D} 10)$ & 63.2 (SK-OV-3) & [35] \\
\hline & \multirow{2}{*}{ Microglossa pyrifolia } & \multirow{2}{*}{$\begin{array}{c}\text { Petroleum ether-EtOAc } \\
(1: 1, v / v)\end{array}$} & E-phytol (20) & $8.5(\mathrm{PoW}) ; 11.5(\mathrm{Dd} 2)$ & - & [36] \\
\hline & & & 6E-geranylgeraniol-19-oic acid (21) & $12.9(\mathrm{PoW}) ; 15.6(\mathrm{Dd} 2)$ & - & \\
\hline & \multirow{2}{*}{ Echinops hoehnelii } & \multirow{2}{*}{$\mathrm{CH}_{2} \mathrm{Cl}_{2}$} & 5-(penta-1,3-diynyl)-2-(3,4-dihydroxybut-1-ynyl)-thiophene (22) & $50.2 \%(100 \mathrm{mg} / \mathrm{kg})$ & - & [37] \\
\hline & & & 5-(penta-1,3-diynyl)-2-(3-chloro-4-acetoxy-but-1-yn)-thiophene (23) & $32.7 \%(100 \mathrm{mg} / \mathrm{kg})$ & - & \\
\hline \multirow{4}{*}{ Buxaceae } & \multirow{4}{*}{ Buxus semperviren } & \multirow{4}{*}{$\mathrm{MeOH}$} & compound (24) & $0.5-3.0$ (HB3) & 7.0 (Hela) & [38] \\
\hline & & & compound (25) & $0.5-3.0(\mathrm{HB} 3)$ & $>20$ (Hela) & \\
\hline & & & 23-O-(trans)-feruloyl-23-hydroxybetulin (26) & $0.5-3.0$ (HB3) & $>20$ (Hela) & \\
\hline & & & compound (27-31) & $0.5-3.0(\mathrm{HB} 3)$ & $>20$ (Hela) & \\
\hline \multirow{2}{*}{ Cecropiaceae } & \multirow{2}{*}{ Cecropia pachystachya } & \multirow{2}{*}{ EtOH } & $\beta$-sitosterol (32) & $>120$ (W2) & - & [39] \\
\hline & & & tormentic acid (33) & 19.0-25.2 (W2) & - & \\
\hline
\end{tabular}


Table 2. Cont.

\begin{tabular}{|c|c|c|c|c|c|c|}
\hline Family & Species & Extract Solvent & Compound & $\begin{array}{c}\text { Antiplasmodial } \mathrm{IC}_{50}(\mu \mathrm{M})^{\text {a }} \\
\quad(\text { P. falciparum })\end{array}$ & $\begin{array}{c}\text { Cytotoxicity, } \mathrm{ED}_{50}(\mu \mathrm{M})^{\mathrm{b}} \\
(\text { Cell Line) }\end{array}$ & References \\
\hline \multirow{32}{*}{ Chloranthaceae } & \multirow{19}{*}{ Chloranthus. fortunei } & \multirow{19}{*}{ EtOH } & fortunilide A (34) & $0.005(\mathrm{Dd} 2)$ & 8.8 (WI-38) & \multirow{32}{*}{ [40] } \\
\hline & & & fortunilide B (35) & 0.02 (Dd2) & 3.1 (WI-38) & \\
\hline & & & fortunilide C (36) & $0.2(\mathrm{Dd} 2)$ & - & \\
\hline & & & fortunilide D (37) & $0.03(\mathrm{Dd} 2)$ & 0.5 (WI-38) & \\
\hline & & & fortunilide E (38) & 0.04 (Dd2) & $>100(\mathrm{WI}-38)$ & \\
\hline & & & fortunilide F (39) & $5.3(\mathrm{Dd} 2)$ & - & \\
\hline & & & fortunilide G (40) & $0.05(\mathrm{Dd} 2)$ & 1.2 (WI-38) & \\
\hline & & & fortunilide $\mathrm{H}$ (41) & $0.2(\mathrm{Dd} 2)$ & - & \\
\hline & & & fortunilide I (42) & 0.09 (Dd2) & - & \\
\hline & & & fortunilide J (43) & $9.9(\mathrm{Dd} 2)$ & - & \\
\hline & & & fortunilide K (44) & $4.7(\mathrm{Dd} 2)$ & - & \\
\hline & & & fortunilide L (45) & $0.1(\mathrm{Dd} 2)$ & 15.5 (WI-38) & \\
\hline & & & sarglabolide I (46) & $4.6(\mathrm{Dd} 2)$ & - & \\
\hline & & & sarglabolide J (47) & $0.007(\mathrm{Dd} 2)$ & 4.0 (WI-38) & \\
\hline & & & shizukaol K (48) & $0.9(\mathrm{Dd} 2)$ & - & \\
\hline & & & shizukaol I (49) & $0.1(\mathrm{Dd} 2)$ & - & \\
\hline & & & shizukaol C (50) & $0.02(\mathrm{Dd} 2)$ & 0.8 (WI-38) & \\
\hline & & & schizukaol M (51) & 0.10 (Dd2) & 4.5 (WI-38) & \\
\hline & & & chlorahololide D (53) & $0.01(\mathrm{Dd} 2)$ & $0.2(\mathrm{WI}-38)$ & \\
\hline & C. multisachys & - & chloramultilide B (65) & $7.1(\mathrm{Dd} 2)$ & - & \\
\hline & \multirow{9}{*}{ C. serratus and C. spicatus } & \multirow{9}{*}{-} & chlorajaponilide C (52) & 0.001 (Dd2) & $5.4(\mathrm{WI}-38)$ & \\
\hline & & & shizukaol N (54) & $0.1(\mathrm{Dd} 2)$ & $10.0(\mathrm{WI}-38)$ & \\
\hline & & & shizukaol E (58) & $1.8(\mathrm{Dd} 2)$ & - & \\
\hline & & & shizukaol D (59) & $0.6(\mathrm{Dd} 2)$ & - & \\
\hline & & & shizukaol F (60) & 0.01 (Dd2) & 0.2 (WI-38) & \\
\hline & & & shizukaol G (61) & $0.01(\mathrm{Dd} 2)$ & 1.7 (WI-38) & \\
\hline & & & shizukaol B (62) & 0.03 (Dd2) & 16.7 (WI-38) & \\
\hline & & & spicachlorantin D (63) & $0.5(\mathrm{Dd} 2)$ & - & \\
\hline & & & shizukaol A (64) & $1.5(\mathrm{Dd} 2)$ & - & \\
\hline & \multirow{3}{*}{ Sarcandra glabra } & \multirow{3}{*}{-} & sarcandrolide B (55) & $0.27(\mathrm{Dd} 2)$ & - & \\
\hline & & & sarcandrolide A (56) & $0.3(\mathrm{Dd} 2)$ & - & \\
\hline & & & sarcandrolide J (57) & $11.4(\mathrm{Dd} 2)$ & - & \\
\hline
\end{tabular}


Table 2. Cont.

\begin{tabular}{|c|c|c|c|c|c|c|}
\hline Family & Species & Extract Solvent & Compound & $\begin{array}{l}\text { Antiplasmodial } \mathrm{IC}_{50}(\mu \mathrm{MM})^{\mathrm{a}} \\
(\text { (P. falciparum) }\end{array}$ & $\begin{array}{l}\text { Cytotoxicity, } \mathrm{ED}_{50}(\mu \mathrm{M})^{\mathrm{b}} \\
\quad \text { (Cell Line) }\end{array}$ & References \\
\hline \multirow{3}{*}{ Chrysobalanceae } & \multirow{3}{*}{ Parinari capensis } & \multirow{3}{*}{$\begin{array}{l}\text { Petroleum ether and } \\
\qquad \mathrm{CH}_{2} \mathrm{Cl}_{2}\end{array}$} & $\begin{array}{l}\text { 10,13-dihydroxy-9-methyl-15-oxo-20-norkaur-16-en-18-oic acid } \\
\gamma \text {-lactone (66) }\end{array}$ & 1.7 (FCR-3) & 5.5 (Graham) & [41] \\
\hline & & & $\begin{array}{l}\text { 10-hydroxy-13-methoxy-9-methyl-15-oxo-20-norkaur-16-en-18-oic } \\
\text { acid } \gamma \text {-lactone (37) }\end{array}$ & 1.9 (FCR-3) & 3.2 (Graham) & \\
\hline & & & $\begin{array}{l}\text { 10-hydroxy-9-methyl-15-oxo-20-norkaur-16-en-18-oic acid } \gamma \text {-lactone } \\
\text { (68) }\end{array}$ & 5.0 (FCR-3) & 9.6 (Graham) & \\
\hline \multirow{5}{*}{ Clusiaceae } & \multirow{5}{*}{ Garcinia mckeaniana } & \multirow{5}{*}{ Acetone } & mckeanianones A (69) & 6.2 (TM4) & - & [42] \\
\hline & & & mckeanianones B (70) & 6.7 (TM4) & 12.9 (Vero) & \\
\hline & & & mckeanianones C (71) & 6.0 (TM4) & 29.5 (Vero) & \\
\hline & & & bannaxanthones I (72) & 8.5 (TM4) & - & \\
\hline & & & bannaxanthones E (73) & 8.3 (TM4) & - & \\
\hline \multirow{3}{*}{ Connaraceae } & \multirow{3}{*}{ Rourea minor (Gaertn.) Aubl. } & \multirow{3}{*}{$\mathrm{CHCl}_{3}$} & rourinoside (74) & 3.7 (D6); 2.1 (W2) & $\mathrm{KB}: \mathrm{ED}_{50}:>35.1$ & [26] \\
\hline & & & rouremin $(75)$ & 5.1 (D6); 4.5 (W2) & $\mathrm{KB}: \mathrm{ED}_{50}:>25.5$ & \\
\hline & & & 1-(26-hydroxyhexacosanoyl)-glycerol (76) & 9.5 (D6); 12.7 (W2) & $\mathrm{KB}: \mathrm{ED}_{50}:>41.2$ & \\
\hline \multirow{4}{*}{ Cornaceae } & \multirow{4}{*}{ Cornus florida $\mathrm{L}$. } & \multirow{4}{*}{ EtOH } & ergosta-4,6,8,22-tetraene-3-one (77) & $61.0(\mathrm{D} 10)$ & $27.0(\mathrm{~L} 6)$ & [43] \\
\hline & & & 3-epideoxyflindissol (78) & $128.0(\mathrm{D} 10)$ & 14.7 (L6) & \\
\hline & & & $3 \beta$-O-cis-coumaroyl betulinic acid (79) & 10.4 (D10) & 5.6 (L6) & \\
\hline & & & $3 \beta$-O-trans-coumaroyl betulinic acid (80) & $15.3(\mathrm{D} 10)$ & 9.3 (L6) & \\
\hline \multirow{3}{*}{ Cucurbitaceae } & \multirow{3}{*}{ Cogniauxia podolaena Baill. } & \multirow{3}{*}{$\mathrm{CH}_{2} \mathrm{Cl}_{2}$} & cucurbitacin B (81) & 2.9 (FcM29 strain) & $94 \%$ inhibition of $\mathrm{KB}$ at $1.8 \mu \mathrm{M}$ & [44] \\
\hline & & & cucurbitacin D (82) & 7.8 (FcM29 strain) & $95 \%$ inhibition of $\mathrm{KB}$ at $1.9 \mu \mathrm{M}$ & \\
\hline & & & 20-epibryonolic acid (83) & 4.4 (FcM29 strain) & $20 \%$ inhibition of $\mathrm{KB}$ at $2.2 \mu \mathrm{M}$ & \\
\hline Ebenaceae & Diospyros quaesita Thw. & $\mathrm{CHCl}_{3}$ & betulinic acid 3-caffeate (84) & $1.4(\mathrm{D} 6) ; 1.0(\mathrm{~W} 2)$ & $4.0(\mathrm{~KB})$ & [27] \\
\hline \multirow{4}{*}{ Euphorbiaceae } & \multirow{2}{*}{ Jatropha isabelli } & \multirow{2}{*}{-} & compound 85 & - & - & [45] \\
\hline & & & compound $\mathbf{8 6}$ & - & - & \\
\hline & \multirow{2}{*}{ Strophioblachia fimbricalyx } & \multirow{2}{*}{$\mathrm{MeOH}$} & 9-O-demethyltrigonostemone (87) & 8.7 (K1) & $2.6(\mathrm{~KB})$ & [46] \\
\hline & & & 3,6,9-trimethoxyphenanthropolone (88) & 9.9 (K1) & $12.3(\mathrm{~KB})$ & \\
\hline \multirow{5}{*}{ Fabaceae } & Cajanus cajan $\mathrm{L}$. & - & cajachalcone $(\mathbf{8 9})$ & $7.4(\mathrm{~K} 1)$ & - & [47] \\
\hline & \multirow{2}{*}{ Piptadenia pervillei } & \multirow{2}{*}{ EtOAc } & (+)-catechin 5-gallate (70) & $1.2(\mathrm{FcB} 1)$ & $>75$ (MRC-5) & [48] \\
\hline & & & (+)-catechin 3-gallate (91) & $1.0(\mathrm{FcB} 1)$ & $>75$ (MRC-5) & \\
\hline & \multirow{2}{*}{$\begin{array}{l}\text { Prosopis glandulosa var. } \\
\text { glandulosa }\end{array}$} & \multirow{2}{*}{$\mathrm{EtOH}$} & prosopilosidine (92) & 0.1 (D6); 0.3 (W2) & $20.2(\mathrm{~KB})$ & [49] \\
\hline & & & isoprosopilosidine (93) & 0.1 (D6); $0.3(\mathrm{~W} 2)$ & $18.8(\mathrm{~KB})$ & \\
\hline Fagaceae & Quercus laceyi & $\mathrm{MeOH}$ & kaempferol 3-O-glucosides (94-97) & $0.6-2.1$ (HB3) & $<3.0$ (Hela) & [38] \\
\hline \multirow{3}{*}{ Hypericaceae } & Vismia orientalis & - & vismione $\mathrm{D}(98)$ & $2.4(\mathrm{~K} 1)$ & 10.0 (L6 cell) & [50] \\
\hline & \multirow{2}{*}{ Psorospermum glaberrimum } & \multirow{2}{*}{ Hexane } & 3-geranyloxyemodin anthrone (99) & 1.7 (W2) & - & [51] \\
\hline & & & acetylvismione D (100) & 0.1 (W2) & - & \\
\hline
\end{tabular}


Table 2. Cont

\begin{tabular}{|c|c|c|c|c|c|c|}
\hline Family & Species & Extract Solvent & Compound & $\begin{array}{l}\text { Antiplasmodial } \mathrm{IC}_{50}(\mu \mathrm{M})^{\mathrm{a}} \\
\quad(\text { P. falciparum })\end{array}$ & $\begin{array}{c}\text { Cytotoxicity, } \mathrm{ED}_{50}(\mu \mathrm{M})^{\mathrm{b}} \\
(\text { Cell Line) }\end{array}$ & References \\
\hline \multirow{5}{*}{ Lamiaceae } & Ocimum sanctum & EtOAc & compound 101 & $0.1(3 \mathrm{DD} 7)$ & - & [52] \\
\hline & \multirow{2}{*}{ Phlomis brunneogaleata } & \multirow{2}{*}{$\mathrm{MeOH}$} & luteolin 7-O- $\beta$-D-glucopyranoside (102) & $5.4(\mathrm{~K} 1)$ & $>200$ & [53] \\
\hline & & & chrysoeriol 7-O- $\beta$-D-glucopyranoside (103) & $12.7(\mathrm{~K} 1)$ & $>194$ & \\
\hline & \multirow{2}{*}{ Salvia radula } & \multirow{2}{*}{$\mathrm{MeOH}: \mathrm{CHCl}_{3}=1: 1$} & betulafolientriol oxide (104) & 10.4 (FCR-3) & - & [54] \\
\hline & & & salvigenin (105) & 75.0 (FCR-3) & 207 (MCF-7) & \\
\hline \multirow{2}{*}{ Loganiaceae } & \multirow{2}{*}{ Strychnos icaja } & \multirow{2}{*}{$\begin{array}{l}\text { EtOAc-EtOH-NH }{ }_{4} \mathrm{OH} \\
\qquad(96: 3: 1)\end{array}$} & 15-hydroxyvomicine (106) & $101.0(\mathrm{~W} 2)$ & - & [55] \\
\hline & & & N-methyl-sec-iso-pseudostrychnine (107) & 110.6 (W2) & - & \\
\hline \multirow{3}{*}{ Lythraceae } & \multirow{3}{*}{$\begin{array}{l}\text { Ammannia multiflora, } A \text {. } \\
\text { baccifera }\end{array}$} & \multirow{3}{*}{$\mathrm{MeOH}$} & 4-hydroxy- $\alpha$-tetralone (108) & 194.0 (NF-54) & - & [56] \\
\hline & & & tetralone-4-O- $\beta$-D-glucopyranoside (109) & 124.0 (NF-54) & - & \\
\hline & & & ammaniol (110) & 88.3 (NF-54) & - & \\
\hline Malvaceae & Thespesia danis. & Acetone-water (7:3) & (R)-(-)-gossypol (111) & $4.5(3 \mathrm{D} 7)$ & - & [57] \\
\hline \multirow{3}{*}{ Monimiaceae } & Doryphora sassafras & $\mathrm{CH}_{2} \mathrm{Cl}_{2} / \mathrm{MeOH}$ & $\begin{array}{l}\text { 1-(4-hydroxybenzyl)-6,7-methylenedioxy-2-methylisoquinolinium } \\
\text { trifluoroacetate (112) }\end{array}$ & 3.0 (3D7); $4.4(\mathrm{Dd} 2)$ & 120.0 (HEK293) & [58] \\
\hline & \multirow[t]{2}{*}{ Glossocalyx brevipes Benth. } & \multirow[t]{2}{*}{$\mathrm{CHCl}_{3} / \mathrm{MeOH}(1 / 1)$} & $\begin{array}{c}\text { methyl 2-(1' } \beta \text {-geranyl-5' } \beta \text {-hydroxy-2'-oxocyclohex-3'-enyl) acetate } \\
\text { (113) }\end{array}$ & $2.2(\mathrm{D} 6) ; 6.6(\mathrm{~W} 2)$ & - & [59] \\
\hline & & & 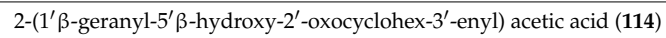 & 4.8 (D6); 8.3 (W2) & - & \\
\hline \multirow{4}{*}{ Moraceae } & Ficus fistulosa & - & verrucarin $L$ acetate $(4)$ & $0.001(\mathrm{D} 6) ; 0.001(\mathrm{~W} 2)$ & $0.2(\mathrm{~KB})$ & [24] \\
\hline & \multirow{3}{*}{ F. septica } & \multirow{3}{*}{$\mathrm{MeOH}$} & dehydrotylophorine (115) & $0.4(3 \mathrm{D} 7)$ & 8.2 (L929) & [60] \\
\hline & & & dehydroantofine (116) & $0.03(3 \mathrm{D} 7)$ & $>55$ (L929) & \\
\hline & & & tylophoridicine D (117) & $0.06(3 \mathrm{D} 7)$ & $>56$ (L929) & \\
\hline Myristicaceae & Knema glauca & EtOAc & malabaricone A (118) & 8.5 (K1) & $>61$ (KB); 55.4 (NCI-H187) & [61] \\
\hline \multirow{5}{*}{ Piperaceae } & \multirow{2}{*}{ Piper sarmentosum } & \multirow{2}{*}{ Hexane-MeOH } & sarmentine (119) & 85.5 (K1) & - & [62] \\
\hline & & & 1-piperettyl pyrrolidine (120) & $21.9(\mathrm{~K} 1)$ & - & \\
\hline & \multirow{3}{*}{ P. tricuspe } & \multirow{3}{*}{ Petroleum ether } & dictyochromenol (121) & 9.6 (FcB1) & $7.7(\mathrm{~L}-6)$ & [63] \\
\hline & & & 3-farnesyl-p-hydroxy benzoic acid (122) & 29.8 (FcB1) & 40.9 (L-6) & \\
\hline & & & 2'E,6'E 2-farnesyl hydroquinone (123) & 1.4 (FcB1) & $1.1(\mathrm{~L}-6)$ & \\
\hline Platanaceae & Platanus occidentalis & $\mathrm{MeOH}$ & kaempferol 3-O-rhamnosides (124-127) & $0.5-1.8$ (HB3) & 9.3-20.0 (Hela) & [38] \\
\hline \multirow{4}{*}{ Rubiaceae } & \multirow{4}{*}{ Nauclea orientalis } & \multirow{4}{*}{$\mathrm{MeOH}$} & $\begin{array}{l}\text { naucleaorine (128) } \\
\end{array}$ & 6.9 (D6); 8.0 (W2) & $38.0(\mathrm{~KB})$ & [28] \\
\hline & & & epimethoxynaucleaorine (129) & 12.4 (D6); 13.2 (W2) & $>37.9(\mathrm{~KB})$ & \\
\hline & & & 3x,23-dihydroxyurs-12-en-28-oic acid (130) & 9.7 (D6); 12.7 (W2) & $>42.2$ (KB) & \\
\hline & & & oleanolic acid (131) & 4.6 (D6); $5.1(\mathrm{~W} 2)$ & $46.0(\mathrm{~KB})$ & \\
\hline
\end{tabular}


Table 2. Cont.

\begin{tabular}{|c|c|c|c|c|c|c|}
\hline Family & Species & Extract Solvent & Compound & $\begin{array}{c}\text { Antiplasmodial } \mathrm{IC}_{50}(\mu \mathrm{MM})^{\mathrm{a}} \\
(\text { (P. falciparum) }\end{array}$ & $\begin{array}{c}\text { Cytotoxicity, } \mathrm{ED}_{50}(\mu \mathrm{M})^{\mathrm{b}} \\
\text { (Cell Line) }\end{array}$ & References \\
\hline \multirow{5}{*}{ Rutaceae } & \multirow{2}{*}{ Citropsis articulata } & \multirow{2}{*}{$\mathrm{MeOH}$} & 5-hydroxynoracronycine (132) & $2.8(\mathrm{FcB} 1)$ & 28.8 (Vero) & [64] \\
\hline & & & 1,5-dihydroxy-2,3-dimethoxy-10-methyl-9-acridone (133) & 10.0 (FcB1) & 101 (Vero) & \\
\hline & \multirow{3}{*}{$\begin{array}{l}\text { Zanthoxylum chiloperone var. } \\
\text { angustifolium Engl. }\end{array}$} & \multirow{3}{*}{$\mathrm{CH}_{2} \mathrm{Cl}_{2}$} & trans-avicennol (134) & $\begin{array}{c}7.8 \text { (K1); } 1.5 \text { (F32); } 3.5 \text { (PFB); } 6.4 \\
\text { (FcB1) }\end{array}$ & 12.8 (MCR5) & \\
\hline & & & canthin-6-one (135) & $\begin{array}{c}24.1 \text { (K1); 9.1 (F32); } 14.6 \text { (PFB); } \\
\text { 18.2 (FcB1) }\end{array}$ & 42.7 (MCR5) & [65] \\
\hline & & & 5-methoxycanthin-6-one (136) & $20.4(\mathrm{~K} 1) ; 41.6$ (F32) & - & \\
\hline \multirow{3}{*}{ Simaroubaceae } & \multirow{2}{*}{ Eurycoma longifolia } & \multirow{2}{*}{$\mathrm{CH}_{2} \mathrm{Cl}_{2}$} & eurycomanone (137) & 0.06 (D6); $0.04(\mathrm{~W} 2)$ & 0.02 (A-549); <0.006 (MCF-7) & {$[66,67]$} \\
\hline & & & pasakbumin B (138) & 0.08 (D6); $0.05(\mathrm{~W} 2)$ & 0.02 (A-549); <0.006 (MCF-7) & \\
\hline & Picrolemma sprucei & Hexane $/ \mathrm{H}_{2} \mathrm{O}$ & neosergeolide (139) & $0.002(\mathrm{~K} 1)$ & - & [68] \\
\hline \multirow{2}{*}{ Apocynaceae } & Aspidosperma vargasii & EtOH & ellipticine (140) & $0.07(\mathrm{~K} 1)$ & - & \\
\hline & A. desmanthum & EtOH & aspidocarpine (141) & $0.02(\mathrm{~K} 1)$ & - & \\
\hline Piperaceae & Pothomorphe peltata & $\mathrm{CHCl}_{3} / \mathrm{EtOH}$ & 4-nerolidylcatechol (142) & 0.7 (K1) & - & \\
\hline \multirow{2}{*}{ Theaceae } & \multirow{2}{*}{ Camellia sinensis } & & mefloquine (143) & - & - & [69] \\
\hline & & & gallocatecin (144) & - & - & \\
\hline \multirow{5}{*}{ Tiliaceae } & \multirow{5}{*}{ Grewia bilamellata } & \multirow{5}{*}{$\mathrm{MeOH}$} & 3 $\alpha, 20$-lupandiol (145) & 19.8 (D6); 19.1 (W2) & $>90(\mathrm{~KB})$ & [29] \\
\hline & & & grewin (146) & 11.2 (D6); 5.5 (W2) & $>107.5$ (KB) & \\
\hline & & & nitidanin (147) & 21.2 (D6); 18.4 (W2) & $>90(\mathrm{~KB})$ & \\
\hline & & & $2 \alpha, 3 \beta$-dihydroxyolean-12-en-28-oic acid (148) & 21.1 (D6); 8.6 (W2) & $51.5(\mathrm{~KB})$ & \\
\hline & & & 2,6-dimethoxy-1-acetonylquinol (149) & 42.2 (D6); $23.0(\mathrm{~W} 2)$ & 169 (KB) & \\
\hline Verbenaceae & Lippia javanica & EtOAc (aerial parts) & lippialactone (150) & 23.8 (D10) & - & [70] \\
\hline
\end{tabular}

${ }^{\mathrm{a}} \mathrm{IC}_{50}$ : Concentration that resulted in 50\% death of Plasmodium falciparum. ${ }^{\mathrm{b}} \mathrm{ED}_{50}$ : Concentration that resulted in $50 \%$ cell death. 
Table 3. The ethnology of plants.

\begin{tabular}{|c|c|c|c|c|c|c|}
\hline Family & Ethnologic Plant & Country & Plant Part & $\begin{array}{l}\text { Antiplasmodial Activity }\left(\mathrm{IC}_{50}\right) \\
(\mu \mathrm{g} / \mathrm{mL}, \text { Unless Indicated })^{\mathrm{a}} \\
(\text { P. falciparum })\end{array}$ & $\begin{array}{l}\text { Cytotoxicity }\left(\mathrm{CC}_{50} \text { for Cells, } \mathrm{LD}_{50}\right. \\
\text { for Brine Shrimp) ( } \mathrm{gg} / \mathrm{gL} \mathrm{h}, \text { Unless } \\
\text { Indicated })^{, \mathrm{c}} \\
\text { (Cell Line) }\end{array}$ & References \\
\hline Acanthaceae & $\begin{array}{l}\text { Justilia schimperand } \\
\text { (Hochst ex Nees) T. Alnder }\end{array}$ & & Roots & - & - & [71] \\
\hline \multirow{7}{*}{ Anacardiaceae } & \multirow{3}{*}{ Mangifera indica $\mathrm{L}$. } & Africa & Leaves & $\begin{array}{l}\% \text { parasitaemia reduced from } 8.9 \text { at } \\
60 \mathrm{mg} / \mathrm{kg} \text { to } 7.2 \text { at } 240 \mathrm{mg} / \mathrm{kg} \text { (mice) }\end{array}$ & $208.3 \mathrm{mg} / \mathrm{kg}$ (mice) & [72] \\
\hline & & Nigeria & Leaves & - & 3079.1 (brine shrimp) & [73] \\
\hline & & Nigeria & Stem barks & - & 2456.0 (brine shrimp) & {$[73,74]$} \\
\hline & Pseudoprotorhus longifolius H. Perr. & Madagascar & Leaves & - & - & [75] \\
\hline & Rhus taratana (Bak.) H. Perr. & Madagascar & Leaves & - & - & [75] \\
\hline & Sclerocarya birrea (A. Rich) Hochst. & South Africa & Stem-bark (MeOH) & 5.91 (D6) & - & [76] \\
\hline & S. caffra Sond. & Madagascar & Leaves & - & - & [75] \\
\hline \multirow{2}{*}{ Annonaceae } & Annona senegalensis Rolyns \&Gh & Nigeria & Leaves & - & 6811.0 (brine shrimp) & [73] \\
\hline & Enantia chlorantha Oliv. & Nigeria & Stem barks & - & 214.3 (brine shrimp) & {$[73,74]$} \\
\hline \multirow{14}{*}{ Apocynaceae } & Alstonia boonei DeWild & Nigeria & Leaves; stem barks & $\begin{array}{l}\text { \% parasitaemia reduced from } 19.4 \% \\
\text { (negative control) to } 5.5 \% \text { at } 240 \\
\mathrm{mg} / \mathrm{kg} \text { (mice) }\end{array}$ & $78.77 \mathrm{mg} / \mathrm{kg}$ (mice) & {$[72,74]$} \\
\hline & Aspidosperma cylindrocarpon Müll. Arg. & Brazil & Trunk woods (EtOH) & 44.0 (W2); 39.0 (3D7) & $>500$ (Vero) & [7] \\
\hline & A. parvifolium A. DC. & Brazil & Trunk barks (EtOH) & 32.8 (W2); 20.5 (3D7) & $>500$ (Vero) & [7] \\
\hline & \multirow{5}{*}{ A. olivaceum Müll. Arg. } & \multirow{5}{*}{ Brazil } & Leaves $\left(\mathrm{CH}_{2} \mathrm{Cl}_{2}\right)$ & 7.0 (W2); 25.5 (3D7) & $>500$ (Vero) & [7] \\
\hline & & & Leaves (EtOH) & 7.0 (W2); 5.0 (3D7) & - & \\
\hline & & & Trunk wood $\left(\mathrm{CH}_{2} \mathrm{Cl}_{2}\right)$ & $<6(\mathrm{~W} 2) ;<6(3 \mathrm{D} 7)$ & $>500$ (Vero) & \\
\hline & & & Trunk bark $\left(\mathrm{CH}_{2} \mathrm{Cl}_{2}\right)$ & $<6(\mathrm{~W} 2) ;<6(3 \mathrm{D} 7)$ & - & \\
\hline & & & Trunk bark (EtOH) & 5.0 (W2); 7.0 (3D7) & $>500$ (Vero) & \\
\hline & \multirow{6}{*}{ A. ramiflorum Müll. Arg. } & \multirow{6}{*}{ Brazil } & Leaves (EtOH) & 32.8 (W2); 20.5 (3D7) & - & [7] \\
\hline & & & Leaves $\left(\mathrm{CH}_{2} \mathrm{Cl}_{2}\right)$ & $<6$ (W2); <6 (3D7) & - & \\
\hline & & & Trunk woods (EtOH) & 36.5 (W2); 48.0 (3D7) & - & \\
\hline & & & Trunk woods $\left(\mathrm{CH}_{2} \mathrm{Cl}_{2}\right)$ & $9.5(3 \mathrm{D} 7)$ & $>500$ (Vero) & \\
\hline & & & Trunk woods (EtOH) & 19.8 (W2); 1.0 (3D7) & - & \\
\hline & & & Trunk barks $\left(\mathrm{CH}_{2} \mathrm{Cl}_{2}\right)$ & $<6(\mathrm{~W} 2) ;<6(3 \mathrm{D} 7)$ & $>500$ (Vero)) & \\
\hline
\end{tabular}


Table 3. Cont

\begin{tabular}{|c|c|c|c|c|c|c|}
\hline Family & Ethnologic Plant & Country & Plant Part & $\begin{array}{l}\text { Antiplasmodial Activity }\left(\mathrm{IC}_{50}\right) \\
(\mu \mathrm{g} / \mathrm{mL} \text {, Unless Indicated })^{\mathrm{a}} \\
(\text { P. falciparum })\end{array}$ & $\begin{array}{l}\text { Cytotoxicity }\left(\mathrm{CC}_{50} \text { for Cells, } \mathrm{LD}_{50}\right. \\
\text { for Brine Shrimp) }(\mu \mathrm{gg} / \mathrm{mL}, \text { Unless } \\
\text { Indicated })^{, \mathrm{c}} \\
\text { (Cell Line) }\end{array}$ & References \\
\hline & \multirow{8}{*}{ A. spruceanum Benth. ex Müll. Arg. } & \multirow{8}{*}{ Brazil } & Leaves (EtOH) & 65.0 (W2); >100 (3D7) & - & [7] \\
\hline & & & Leaves $\left(\mathrm{CH}_{2} \mathrm{Cl}_{2}\right)$ & 23.25 (W2); 47.0 (3D7) & - & \\
\hline & & & Trunk woods (EtOH) & 29.5 (W2); 41.5 (3D7) & - & \\
\hline & & & Trunk woods $\left(\mathrm{CH}_{2} \mathrm{Cl}_{2}\right)$ & $<6(\mathrm{~W} 2) ;<6$ (3D7) & 109.6 (Vero)) & \\
\hline & & & Trunk woods $\left(\mathrm{CHCl}_{3}\right)$ & 37.0 (W2); >100 (3D7) & - & \\
\hline & & & Trunk barks (EtOH) & 26.3 (W2); 14.0 (3D7) & - & \\
\hline & & & Trunk barks $\left(\mathrm{CH}_{2} \mathrm{Cl}_{2}\right)$ & $<6$ (W2); <6 (3D7) & - & \\
\hline & & & Trunk barks (EłOH) & 28.0 (W2); 19.0 (3D7) & - & \\
\hline & \multirow{4}{*}{ A. tomentosum Mart. } & \multirow{4}{*}{ Brazil } & Trunk woods (EtOH) & $26.5(\mathrm{~W} 2) ; 25.0(3 \mathrm{D} 7)$ & - & {$[7]$} \\
\hline & & & Leaves (EtOH) & 23.8 (W2); 27.0 (3D7) & - & \\
\hline & & & Fruits (EtOH) & 20.5 (W2); 38.6 (3D7) & - & \\
\hline & & & Seeds (EtOH) & 24.5 (W2); 3.0 (3D7) & $>500$ (Vero)) & \\
\hline Aristolochiaceae & Aristolochia acuminata Lamk. & Madagascar & Roots, stems, leaves & - & - & [75] \\
\hline \multirow{3}{*}{ Asteraceae } & Artemisia aпnиа L. & China & Whole plants & - & - & [77] \\
\hline & Tithonia diversifolia A. Gray & Nigeria & Leaves & - & 2304 (brine shrimp) & [73] \\
\hline & Vernonia amygdalina Del. & & Leaves & - & - & [71] \\
\hline \multirow{2}{*}{ Avicenniaceae } & Avicennia marina (Forsk) Vierh. & Madagascar & Aerial parts & - & - & [78] \\
\hline & A. basilicum $\mathrm{L}$. & Madagascar & Aerial parts & - & - & [75] \\
\hline \multirow{2}{*}{ Bignoniaceae } & Fernandoa sp. & Madagascar & Aerial parts & - & - & [75] \\
\hline & $\begin{array}{l}\text { Kigelianthe madagascariensis Sprague var. } \\
\text { hidebrandtii }\end{array}$ & Madagascar & Leaves & - & - & [75] \\
\hline Brassicaceae & Brassica nigra (L.) Koch. & & Seeds & - & - & [71] \\
\hline Caricaceae & Carica papaya L. & & Leaves, fruits, roots & & - & {$[71,79]$} \\
\hline Celastraceae & Maytenus acuminata (L.f.) Loes & Kenya & leaves, root barks & $36.6-41.5 \%$ & - & [80] \\
\hline \multirow{3}{*}{ Combretaceae } & Combretu raimbaulti Heckel & Madagascar & Leaves & & - & [75] \\
\hline & Terminalia catappa & Nigeria & Leaves (EtOAc) & $3.1(\mathrm{~K} 1)$ & $159.9 \mu \mathrm{g} / \mathrm{L}(\mathrm{L} 6)$ & [81] \\
\hline & T. latifolia Engl. & Nigeria & leaves & - & 272.9 (brine shrimp) & [73] \\
\hline Commelinaceae & Commelina benghalensis $\mathrm{L}$. & Madagascar & Aerial parts & - & - & {$[75]$} \\
\hline
\end{tabular}


Table 3. Cont

\begin{tabular}{|c|c|c|c|c|c|c|}
\hline Family & Ethnologic Plant & Country & Plant Part & $\begin{array}{l}\text { Antiplasmodial Activity }\left(\mathrm{IC}_{50}\right) \\
(\mu \mathrm{g} / \mathrm{mL}, \text { Unless Indicated })^{\mathrm{a}} \\
(\text { P. falciparum })\end{array}$ & $\begin{array}{l}\text { Cytotoxicity }\left(\mathrm{CC}_{50} \text { for Cells, } \mathrm{LD}_{50}\right. \\
\text { for Brine Shrimp) (ug/mL, Unless } \\
\text { Indicated) })^{b, c} \\
\text { (Cell Line) }\end{array}$ & References \\
\hline \multirow{14}{*}{ Compositae } & Brachylaena ramiflora (DC.) H. Humb & Madagascar & Aerial parts & - & - & [75] \\
\hline & Conyza aegytiaca Ait. Var lineariloba & Madagascar & Aerial parts & - & - & [75] \\
\hline & Inula perrieri $\mathrm{H}$. Humb. & Madagascar & Leaves & - & - & [75] \\
\hline & Parthenium hysterophorus L. & Madagascar & Aerial parts & - & - & [75] \\
\hline & Senecio ompricaefolius (ex DC.) H. Humb. & Madagascar & Aerial parts & - & - & [75] \\
\hline & Stenocline inuloides DC. & Madagascar & Leaves & - & - & [75] \\
\hline & Tagetes erecta L. & Madagascar & Leaves & - & - & [75] \\
\hline & T. patula L. & Madagascar & Leaves & - & - & [75] \\
\hline & Vernonia lasiopus $\mathrm{O}$. Hoffm. & Kenya & Root barks & - & - & [75] \\
\hline & V. pectoralis Bak. & Madagascar & Aerial parts & - & - & [75] \\
\hline & V. trichodesma Bak. & Madagascar & Leaves & - & - & [75] \\
\hline & V. chapelieri Drak. & Madagascar & Aerial parts & - & - & [75] \\
\hline & V. sp. (Dr. Hely) & Madagascar & Aerial parts & - & - & [75] \\
\hline & V. ampandrandavensis Bak. & Madagascar & Aerial parts & - & - & {$[75]$} \\
\hline \multirow{2}{*}{ Cucurbitaceae } & Momordica charantia $\mathrm{L}$. & Madagascar & Aerial parts & - & - & [75] \\
\hline & Zehneria scabra (Lf.) Sond. & & Roots & - & - & [71] \\
\hline \multirow{15}{*}{ Euphorbiaceae } & Bridelia micrantha Benth. & Nigeria & Leaves & - & $>90,000$ (brine shrimp) & [73] \\
\hline & Croton goudoti $\mathrm{H}$. Bn. & Madagascar & Leaves & - & - & [75] \\
\hline & C. macrostachyus Hochst. Ex Del. & & Leaves/barks/roots & - & - & [71] \\
\hline & Euphorbia hirta & Nigeria & Whole plants (Hexane) & $4.3(\mathrm{~K} 1)$ & 14.2 (L6) & {$[81,82]$} \\
\hline & Flueggea microcarpa Blume & Madagascar & Aerial parts & & - & [75] \\
\hline & \multirow{2}{*}{ Jatropha curcas L. } & Nigeria & Leaves (EtOAc) & $2.4(\mathrm{~K} 1)$ & 126.5 (L6) & {$[75,81,82]$} \\
\hline & & Madagascar & leaves, roots & & & \\
\hline & Manihot utilisma Pohl. & Madagascar & Leaves & - & - & [75] \\
\hline & \multirow{6}{*}{ Phyllanthus amarus Schum. \& Thonn. } & \multirow{2}{*}{$\begin{array}{l}\text { Brazil, Cuba, Haiti, Nigeria, } \\
\text { Elsewhere }\end{array}$} & Whole plants (MeOH) & $5.0(3 \mathrm{D} 7)$ & - & {$[83,84]$} \\
\hline & & & Whole plants $\left(\mathrm{CH}_{2} \mathrm{Cl}_{2}\right)$ & $14.5(3 \mathrm{D} 7)$ & - & \\
\hline & & India & Whole plants & - & - & [85] \\
\hline & & Nigeria & Leaves (EtOAc) & $5.6(\mathrm{~K} 1)$ & 77.7 (L6) & {$[81,82]$} \\
\hline & & Ghana & Whole plants & - & - & {$[85]$} \\
\hline & & West Africa & Aerial parts & - & - & \\
\hline & Phyllanthus sp. & Madagascar & Aerial parts & - & - & [75] \\
\hline
\end{tabular}


Table 3. Cont

\begin{tabular}{|c|c|c|c|c|c|c|}
\hline Family & Ethnologic Plant & Country & Plant Part & $\begin{array}{l}\text { Antiplasmodial Activity }\left(\mathrm{IC}_{50}\right) \\
(\mu \mathrm{g} / \mathrm{mL}, \text { Unless Indicated })^{\mathrm{a}} \\
\text { (P. falciparum) }\end{array}$ & $\begin{array}{l}\text { Cytotoxicity }\left(\mathrm{CC}_{50} \text { for Cells, } \mathrm{LD}_{50}\right. \\
\text { for Brine Shrimp) ( } \mu \mathrm{g} / \mathrm{mL}, \text { Unless } \\
\text { Indicated) } b, c \\
\text { (Cell Line) }\end{array}$ & References \\
\hline \multirow{8}{*}{ Fabaceae } & Acacia nilotica $\mathrm{L}$. & Pakistan & Leaves (EtOH) & $1.3(3 \mathrm{D} 7)$ & - & [86] \\
\hline & Caesalpinia benthamiana & Guinea & Leaves $(\mathrm{MeOH})$ & 4.0 (Ghana) & 32.0 (MRC-5) & [79] \\
\hline & Cajanus cajan Mill sp. & Nigeria & Leaves & - & 988.5 (brine shrimp) & {$[73,74]$} \\
\hline & Calliandra haematocephala Hassk & Nigeria & Roots & - & - & \\
\hline & Calpurna ourea (Ait.) Benth & & Leaves & - & - & [71] \\
\hline & \multirow[t]{2}{*}{ Cassia siamea } & \multirow[t]{2}{*}{ Nigeria } & Stem barks (EtOAc) & $2.70(\mathrm{~K} 1)$ & $\begin{array}{c}988.5 \text { (stem bark), } 8232.2 \text { (brine } \\
\text { shrimp) }\end{array}$ & [73] \\
\hline & & & leaves & - & & \\
\hline & Piliostigma thonnigii Schum & Nigeria & Leaves & - & 7958.0 (brine shrimp) & [73] \\
\hline Flacourtiaceae & Homalium sp. & Madagascar & Aerial parts & - & - & [75] \\
\hline Gramineae & Phragmites mauritianus Kunth & Madagascar & Aerial parts & - & - & [75] \\
\hline Hydrengeaceae & Dichroa febrifuga & China & Roots & - & - & [87] \\
\hline Icacinaceae & $\begin{array}{l}\text { Cassinopsis madagascariensis (Baill.) H. } \\
\text { Bn. }\end{array}$ & Madagascar & Leaves, stem barks & - & - & [75] \\
\hline \multirow{6}{*}{ Lamiaceae } & Hyptispectinata Poit. & Madagascar & Leaves & - & - & [75] \\
\hline & \multirow{2}{*}{ Осітит сапит Sims. } & Nigeria & Leaves (EtOAc) & $1.8(\mathrm{~K} 1)$ & 60.1 (L6) & {$[75,81]$} \\
\hline & & Madagascar & Stems, seeds & - & & \\
\hline & $\begin{array}{l}\text { O. lamiifolium } \\
\text { Hochst. ex Benth. }\end{array}$ & & Leaves & - & - & [71] \\
\hline & Cassytha filiformis $\mathrm{L}$. & Nigeria & Vines & - & - & [74] \\
\hline & Cinnamomum camphora (L.) Sieb & Madagascar & Leaves & - & - & [75] \\
\hline \multirow{8}{*}{ Leguminosae } & Abrus precatorius $\mathrm{L}$. & Madagascar & Leaves & - & - & [75] \\
\hline & Albizzia lebbek Benth. & Madagascar & Aerial parts & - & - & [75] \\
\hline & Caesalpinia bonducella Fleming & Madagascar & Seeds, roots & - & - & [75] \\
\hline & Cassia occidentalis $\mathrm{L}$. & Madagascar & Aerial parts & - & - & [75] \\
\hline & Crotalaria spinosa Hochst. & Madagascar & Leaves & - & - & [75] \\
\hline & Erythryna indica Lamk. & Madagascar & Aerial parts & - & - & [75] \\
\hline & Piliostigma thonningii & Nigeria & Leaves (EtOAc) & $3.6(\mathrm{~K} 1)$ & 56.1 (L6) & [81] \\
\hline & Pongamia pinnata $\mathrm{L}$. & India & Barks (MeOH) & 11.7 (CQ-sensitive) & $>200$ (THP-1) & [88] \\
\hline Lilliaceae & Allium sativum $\mathrm{L}$. & & Bulbs & - & - & [71] \\
\hline \multirow{3}{*}{ Loganiaceae } & Anthocleista amplexicaulus Bak. & Madagascar & Aerial parts & - & - & [75] \\
\hline & A. rhizophoroides Bak. & Madagascar & Roots, leaves & - & - & [75] \\
\hline & Strychnos mostuoides Leeuwenberg & Madagascar & Aerial parts & - & - & [75] \\
\hline
\end{tabular}


Table 3. Cont

\begin{tabular}{|c|c|c|c|c|c|c|}
\hline Family & Ethnologic Plant & Country & Plant Part & $\begin{array}{c}\text { Antiplasmodial Activity }\left(\mathrm{IC}_{50}\right) \\
(\mu \mathrm{g} / \mathrm{mL}, \text { Unless Indicated })^{\mathrm{a}} \\
(\text { P. falciparum })\end{array}$ & $\begin{array}{c}\text { Cytotoxicity }\left(\mathrm{CC}_{50} \text { for Cells, } \mathrm{LD}_{50}\right. \\
\text { for Brine Shrimp) ( } \mu \mathrm{gg} / \mathrm{mL}, \mathrm{L}, \text { Unless } \\
\text { Indicated b, } \\
\text { (Cell Line) }\end{array}$ & References \\
\hline \multirow{3}{*}{ Malvaceae } & Gossypium arboreum $\mathrm{L}$. & Nigeria & Leaves & - & 94.1 (brine shrimp) & [73] \\
\hline & G. barbadense L. & Nigeria & Leaves & - & 3585.0 (brine shrimp) & [73] \\
\hline & G. hirsitum L. & Nigeria & Leaves & - & 257.2 (brine shrimp) & [73] \\
\hline \multirow[t]{3}{*}{ Meliaceae } & Azadirachta indica A. Juss & Africa & leaves & $\begin{array}{l}\text { The percentage parasitaemia } \\
\text { reduced from } 15.7 \% \text { to } 4.8 \% \text { at } 240 \\
\mathrm{mg} / \mathrm{kg} \text { (in vivo) }\end{array}$ & $140.0 \mathrm{mg} / \mathrm{kg}$ (mice) & [72] \\
\hline & \multirow{2}{*}{ Swietenia macrophylla King } & \multirow{2}{*}{ Indonesia } & Seeds & - & - & [89] \\
\hline & & & Barks & $78 \%$ inhibition at 100 (Indo) & - & [90] \\
\hline Melianthaceae & Bersama abyssinica Fresen. & & Leaves, root barks and stems & - & - & [71] \\
\hline \multirow{11}{*}{ Menispermaceae } & Burasaia australis Sc. Elliot & Madagascar & Root barks & - & - & [75] \\
\hline & B. congesta Decne & Madagascar & Root barks & - & - & [75] \\
\hline & B. gracilis Decne & Madagascar & Root barks & - & - & [75] \\
\hline & Burasaia madagascariensis Thou. & Madagascar & Root barks & - & - & [75] \\
\hline & B. nigrescens R. Cap. & Madagascar & Root barks & - & - & [75] \\
\hline & Chasmanthera uviformis Baill. & Madagascar & Stem barks & - & - & [75] \\
\hline & Cissampelos pareira $\mathrm{L}$. & Madagascar & Roots & - & - & [75] \\
\hline & C. madagascariensis (Baill.) Diels. & Madagascar & Roots & - & - & [75] \\
\hline & Spirospermum penduliflorum Thou. & Madagascar & Roots, stem barks & - & - & [75] \\
\hline & Strychnopsis thouarsii Baill. & Madagascar & Leaves, root barks & - & - & [75] \\
\hline & Triclisia macrocarpa (Baill.) Diels & Madagascar & Root barks, stem barks & - & - & [71] \\
\hline Mimosaceae & $\begin{array}{l}\text { Acacia catechu (L.f.) } \\
\text { Willd. }\end{array}$ & & Leaves & - & - & [71] \\
\hline \multirow{3}{*}{ Moraceae } & Ficus elastica Roxb. ex Hornem. & Cameroon & Roots (MeOH) & 9.5 & - & [91] \\
\hline & F. sur Forssk. & Kenya & Leaves, stem barks, root barks & 34.1-48.4\% Inhibition & - & [80] \\
\hline & F. thonningii Blume & Nigeria & Leaves (Hexane) & 2.7 (NF54); 10.4 (K1) & $>20(\mathrm{~KB})$ & [90] \\
\hline Myrtaceae & Psidium guajava $\mathrm{L}$. & Nigeria & Stem barks & - & 707.2 (brine shrimp) & [72] \\
\hline Ochnaceae & Lophira alata Banks & Nigeria & Leaves (Hexane) & 2.5 (NF54); 2.5 (K1) & $>20(\mathrm{~KB})$ & [90] \\
\hline \multirow{2}{*}{ Papilionaceae } & Pericopsis elata Harms & Nigeria & leaves & - & 601.8 (brine shrimp) & [73] \\
\hline & Pterocarpus osun Craib & Nigeria & Stem barks & - & - & [74] \\
\hline \multirow[b]{2}{*}{ Periplocaceae } & Cryptolepts sanguinolenta & West Africa & Roots & - & 13.9 (MCF7) & [92] \\
\hline & $\begin{array}{l}\text { Parquetina nigrescens (Afz.) } \\
\text { Bullock }\end{array}$ & Nigeria & Root barks & - & - & [74] \\
\hline
\end{tabular}


Table 3. Cont

\begin{tabular}{|c|c|c|c|c|c|c|}
\hline Family & Ethnologic Plant & Country & Plant Part & $\begin{array}{l}\text { Antiplasmodial Activity }\left(\mathrm{IC}_{50}\right) \\
(\mu \mathrm{g} / \mathrm{mL}, \text { Unless Indicated })^{\mathrm{a}} \\
(\text { P. falciparum })\end{array}$ & 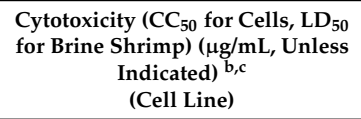 & References \\
\hline Phytolaccacaa & $\begin{array}{l}\text { Phytolacca dodecandra } \\
\text { L'Hér. }\end{array}$ & & Leaves & - & - & {$[71]$} \\
\hline Polygonaceae & Rumex abyssinicus Jacq. & & Leaves and stems & - & - & [71] \\
\hline Potamogetonaceae & Potamogeton javanicus Hass Karl & Madagascar & Aerial parts & - & - & [75] \\
\hline Ranunculaceae & Clematis mauritiana Lamk. Var. normalis & Madagascar & Aerial parts & - & - & [75] \\
\hline \multirow{2}{*}{ Rhamnaceae } & Rhamnus prinoides L' H'erit & Kenya & Leaves, root barks & 34.1-43.9\% Inhibition & - & [80] \\
\hline & R. staddo A. Rich. & Kenya & Root barks & $11.1 \%$ Inhibition & - & [80] \\
\hline \multirow{25}{*}{ Rubiaceae } & Anthospermum emirnense Bak. & Madagascar & Aerial parts & - & - & [75] \\
\hline & Cinchona ledgeriana Muens & Madagascar & Stem barks & - & - & [75] \\
\hline & C. offlcinalis L. & Madagascar & Stem barks & - & - & [75] \\
\hline & C. succirubra Pavon et Kiutzsch & Madagascar & Stem barks & - & - & [75] \\
\hline & Cephalanthus spathelliferus Bak. & Madagascar & Leaves & - & - & [75] \\
\hline & Danais fragrans Gaertn. & Madagascar & Roots & - & - & [75] \\
\hline & D. gerrardii Bak. & Madagascar & Roots & - & - & [75] \\
\hline & D. verticillata Bak. & Madagascar & Roots & - & - & [75] \\
\hline & D. breviflora Bak. & Madagascar & Roots & - & - & [75] \\
\hline & D. cernua Bak. & Madagascar & Roots & - & - & [75] \\
\hline & Hymenodyction lohavato baill. & Madagascar & Root barks, stem barks & - & - & {$[75]$} \\
\hline & \multirow[t]{3}{*}{ Morinda lucida Benth } & Africa & Leaves & $\begin{array}{l}\text { The percentage parasitaemia } \\
\text { reduced from } 14.0 \% \text { to } 5.8 \% \text { at } 240 \\
\mathrm{mg} / \mathrm{kg} \text { (in vivo) }\end{array}$ & $134.5 \mathrm{mg} / \mathrm{kg}$ (mice) & [72] \\
\hline & & Nigeria & Stem barks & P. berghei & 2.6 (brine shrimp) & {$[73]$} \\
\hline & & Nigeria & Leaves & - & 383.9 (brine shrimp) & {$[73]$} \\
\hline & Nauclea latifolia S.M. & Nigeria & Stem barks & - & 9368.0 (brine shrimp) & [73] \\
\hline & Saldinia sp. (andriambavifoy) & Madagascar & Aerial part & - & - & [75] \\
\hline & $\begin{array}{l}\text { Sarcocephalus latifolius } \\
\text { (J. E. Smith) E. A. Bruce }\end{array}$ & Nigeria & Root barks & - & - & {$[74]$} \\
\hline & Schismatoclada concinna Bak. & Madagascar & Root barks & - & - & [75] \\
\hline & S. farahimpensis Bak. & Madagascar & Root barks & - & - & [75] \\
\hline & S. viburnoides Bak. & Madagascar & Root barks & - & - & [75] \\
\hline & \multirow{5}{*}{$\begin{array}{l}\text { Citropsis articulata (Willd. ex Spreng.) } \\
\text { Swingle \& Kellerman }\end{array}$} & Uganda & Roots & $77 \%$ inhibition at 10 (FcB1) & $12 \%$ inhibition at 10 (Vero) & [64] \\
\hline & & & Demethylsuberosin & 16.7 & $>50 \%$ inhibition at 16.7 (Vero) & \\
\hline & & & 5-hydroxynoracronycine & 0.9 & $9.3 \%$ inhibition at 0.9 (Vero) & \\
\hline & & & 1,5-dihydroxy-2,3-dimethoxy & nethyl-9-acridone 3.0 & $30.5 \%$ inhibition at 3.0 (Vero) & \\
\hline & & & $7 \alpha$-obacunyl acetate & 9.3 & $>50 \%$ inhibition at 9.3 (Vero) & \\
\hline
\end{tabular}


Table 3. Cont.

\begin{tabular}{|c|c|c|c|c|c|c|}
\hline Family & Ethnologic Plant & Country & Plant Part & $\begin{array}{c}\text { Antiplasmodial Activity }\left(\mathrm{IC}_{50}\right) \\
(\mu \mathrm{g} / \mathrm{mL}, \text { Unless Indicated })^{\mathrm{a}} \\
(\text { P. falciparum })\end{array}$ & $\begin{array}{l}\text { Cytotoxicity }\left(\mathrm{CC}_{50} \text { for Cells, } \mathrm{LD}_{50}\right. \\
\text { for Brine Shrimp) ( } \mu \mathrm{g} / \mathrm{mL}, \text { Unless } \\
\text { Indicated) }{ }^{b, c} \\
\text { (Cell Line) }\end{array}$ & References \\
\hline \multirow{3}{*}{ Rutaceae } & Evodia fatraina $\mathrm{H}$. Perr & Madagascar & Root barks, stem barks & - & - & [75] \\
\hline & Toddalia asiatica (L.) Lam. & Kenya; Madagascar & $\begin{array}{l}\text { Root barks; root barks, stem } \\
\text { barks }\end{array}$ & - & - & {$[75,80]$} \\
\hline & Zanthoxylum tsihanimpotsa H. Perr. & Madagascar & Stem barks & - & - & [75] \\
\hline Santalaceae & $\begin{array}{l}\text { Okoubaka aubrevillei } \\
\text { Phelleg \& Nomand }\end{array}$ & Nigeria & Stem barks & - & - & [74] \\
\hline Sapindaceae & Dodonaea viscosa Jacq. & Madagascar & Leaves & - & - & [75] \\
\hline Selaginellaceae & Salaginella vogelli & Cameroon & Leaves $(\mathrm{MeOH})$ & 32.2 & - & [91] \\
\hline Schizaeaceae & Mohria caffrorum (L.) Desv. & Madagascar & Aerial parts & - & - & [75] \\
\hline Simaroubaceae & $\begin{array}{l}\text { Brucea antidysenterica } \\
\text { J.F. Mill. }\end{array}$ & & Stems, barks seeds & - & - & [71] \\
\hline \multirow{2}{*}{ Ulmaceae } & Trema commersonii Boj. & Madagascar & Aerial part & - & - & [75] \\
\hline & T. orientalis Blume & Madagascar & Root barks & $2.0(\mathrm{~K} 1)$ & 32.5 (L6) & [75] \\
\hline \multirow{3}{*}{ Verbanaceae } & Lippia multiflora Moldenke & Nigeria & Aerial part & - & 1.1 (brine shrimp) & [73] \\
\hline & Clerodendrum myricoides (Hochst.) Vatke & Kenya & Root barks & 9.8\% (Plasmodium berghei NK65) & - & {$[71,80]$} \\
\hline & Vitex doniana & Nigeria & Leaves (Hexane) & $3.6(\mathrm{~K} 1)$ & 431.4 & {$[81,82]$} \\
\hline \multirow{2}{*}{ Zingiberaceae } & Curcuma longa $\mathrm{L}$. & Madagascar & Leaves & - & - & [75] \\
\hline & Zingiber officinale Roscoe & & Rhizome & - & - & [71] \\
\hline
\end{tabular}

${ }^{\mathrm{a}} \mathrm{IC}_{50}$ : Concentration that resulted in $50 \%$ death of Plasmodium falciparum. ${ }^{\mathrm{b}} \mathrm{LD}_{50}$ : Concentration that was lethal to $50 \%$ of test animals. ${ }^{\mathrm{c}} \mathrm{CC} \mathrm{C}_{50}$ : Concentration that resulted in $50 \%$ cell death. 


\section{Plant-derived Antimalarial Compounds}

\subsection{Annonaceae-Asteraceae Families}

\subsubsection{Annonaceae Family}

Annonaceae is a family of flowering plants consisting of about 2400 species. Two plants in this family have been phytochemically investigated for their antiplasmodial and cytotoxic activities. From the leaves of Friesodielsia discolor, Prawat et al. isolated two new flavonoids, 3'-formyl-2' ,4'-dihydroxy-6'-methoxychalcone (5), 8-formyl-7-hydroxy-5-methoxyflava-none (6), and the known tectochrysin (7) (Figure 3) [31]. They displayed antiplasmodial activity against the K1 multidrug resistant strain of P. falciparum with $\mathrm{IC}_{50}$ values of 9.2, 9.3 and $7.8 \mu \mathrm{M}$, respectively. However, these compounds also exhibited cytotoxicity against the cancer cell lines KB and MCF-7, with the $\mathrm{IC}_{50}$ values ranging from 13.9-34.5 $\mu \mathrm{M}$.<smiles>COc1cc(O)c(C=O)c(O)c1C(=O)/C=C/c1ccccc1</smiles><smiles>O=Cc1c(O)cc(O)c2c1C(=O)C[C@@H](c1ccccc1)O2</smiles>

6<smiles></smiles><smiles>COc1cc(O)c2c(=O)cc(-c3ccccc3)oc2c1</smiles>

7<smiles>COc1ccc2c(c1O)-c1nccc(C)c1C2=O</smiles>

8 $10 \mathrm{R}_{1}=\mathrm{OMe}, \mathrm{R}_{2}=\mathrm{OMe}$

Figure 3. Compounds from Annonaceae plants.

According to Mueller et al. [32], 5-hydroxy-6-methoxyonychine (8), an alkaloid obtained from the roots of the Australian tree plant Mitrephora diversifolia, showed $\mathrm{IC}_{50}$ values of 9.9 and $11.4 \mu \mathrm{M}$ against the 3D7 and Dd2 clones of P. falciparum, respectively.

Miliusacunines A (9) and B (10) were identified from an acetone extract of the leaves and twigs of Miliusa cuneatas [33]. Compound 9 demonstrated inhibitory activity against the TM4 malarial strain ( $\left.\mathrm{IC}_{50} 19.3 \mu \mathrm{M}\right)$, and compound 10 displayed activity against the $\mathrm{K} 1$ malarial strain $\left(\mathrm{IC}_{50} 10.8 \mu \mathrm{M}\right)$. Both isolates showed no toxicity to the Vero cells at the elevated concentrations.

\subsubsection{Araceae Family}

Zhang et al. [22,23] performed extensive research on Rhaphidophora decursiva, a vine growing in Vietnam. The $\mathrm{MeOH}$ extract of the plant leaves and stems showed antimalarial activity against both D6 and W2 clones with no apparent cytotoxicity at a concentration of $20 \mu \mathrm{g} / \mathrm{mL}$. Seven compounds were identified from the stems and leaves of the plant through a bioassay-guided separation (Figure 4). Polysyphorin (1) and rhaphidecurperoxin (2) were among the most active compounds, which demonstrated antimalarial activity with $\mathrm{IC}_{50}$ values of $1.4-1.8 \mu \mathrm{M}$ against the D6 and W2 strains and cytotoxicity with $\mathrm{ED}_{50}$ values of 8.3-13.1 $\mu \mathrm{M}$ against $\mathrm{KB}$ cells (Figure 2). Rhaphidecursinols $\mathrm{A}$ (11) and B (12), grandisin (13), epigrandisin (14) and decursivine (15) also showed activities against P. falciparum (D6 and W2) with $\mathrm{IC}_{50}$ values of 3.4-12.9 $\mu \mathrm{M}$ and cytotoxicity of $\mathrm{ED}_{50}$ values of 23.9-37.0 $\mu \mathrm{M}$ against $\mathrm{KB}$ cells with an exception of compound 14, which showed no antimalarial activity against D6 strain at $23 \mu \mathrm{M}$. 

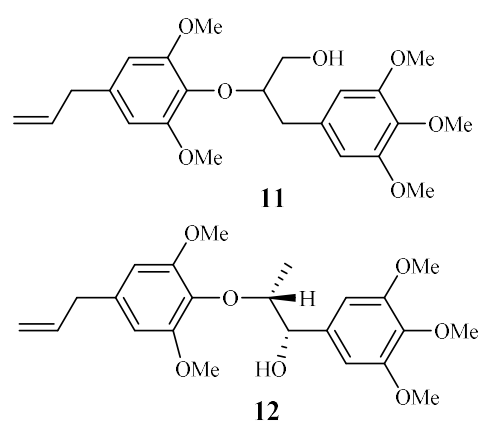

Figure 4. Compounds from an Araceae plant.<smiles>COc1cc(C2O[C@@H](c3cc(OC)c(OC)c(OC)c3)C(C)[C@H]2C)cc(OC)c1OC</smiles><smiles>COc1cc(C2OC(c3cc(OC)c(OC)c(OC)c3)[C@@H](C)C2C)cc(OC)c1OC</smiles><smiles>[14CH2][C@@H]1Oc2ccc3[nH]cc4c3c2[C@@H]1C(=O)NCC4</smiles>

According to the further investigation of Zhang et al. [24], a potent but toxic trichothecene compound, roridin E (3), was identified from the same plant extract (Figure 2). The investigators determined that the compound was able to inhibit parasite growth with $\mathrm{IC}_{50}$ values in the sub-nano molar range. However, roridin $\mathrm{E}$ was also very cytotoxic against KB cells. Interestingly, these researchers reported another trichothecene compound (4) from a plant in a different family, and the compound showed equally potent antimalarial activities as that of roridin E, but with much less cytotoxicity (see Section 2.8.3).

\subsubsection{Asclepiadaceae Family}

Libman et al. reported the antimalarial bioassay-directed separation of Gongronema napalense, leading to the identification of a new steroidal glycoside, gongroneside A (16) (Figure 5) [25]. The compound showed inhibitory activity against the D6 and W2 clones with $\mathrm{IC}_{50}$ values of 1.6 and $1.4 \mu \mathrm{M}$, respectively. Gongroneside A showed no cytotoxicity against KB cells at a concentration of $13.7 \mu \mathrm{M}$.

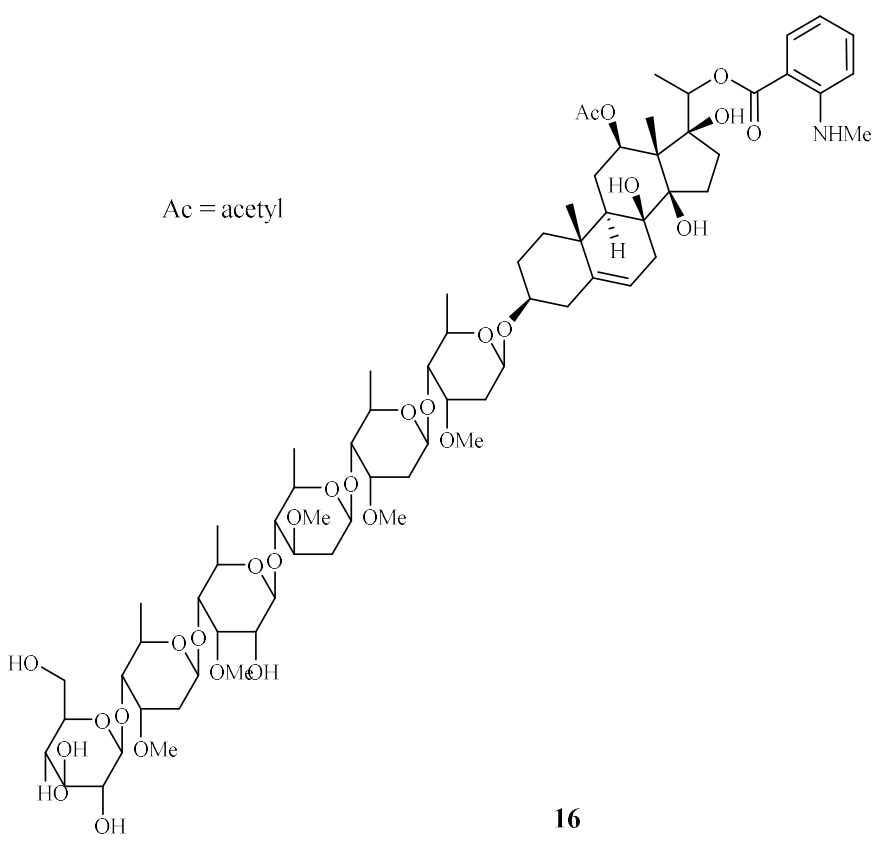

Figure 5. Compound from an Asclepiadaceae plant. 


\subsubsection{Asteraceae Family}

Apigenin 7-O-glucoside (17) and luteolin 7-O-glucoside (18), two flavonoid glycosides obtained from the aerial parts of Achillea millefolium, showed antiplasmodial activities against D10 and W2 strains with $\mathrm{IC}_{50}$ values in the range of 15.3-62.5 $\mu \mathrm{M}$ [34] (Figure 6).<smiles>O=C(O)C(=O)Oc1cc(O)c2c(=O)cc(-c3ccc(O)cc3)oc2c1</smiles>

17<smiles>COc1cc(O)c2c(=O)cc(-c3ccc(O)c(O)c3)oc2c1</smiles>

18<smiles>C=C(C)C1OC(=O)c2cc(C(C)=O)cc(OC)c2O1</smiles>

19<smiles>CC(C)=CCCC(C)=CCCC(=CCCC(C)=CCO)C(=O)O</smiles><smiles>CC#CC#Cc1ccc(C#CC(O)CO)s1</smiles><smiles>CC#CC#Cc1ccc(C#CC(Cl)COC(C)=O)s1</smiles>

Figure 6. Compounds from Asteraceae plants.

2-Isopropenyl-6-acetyl-8-methoxy-1,3-benzodioxin-4-one (19), isolated from the whole plants of the Korean folk medicine Carpesium divaricatum, was reported to show antimalarial activity $[35,93]$ (Figure 6). The compound exhibited activity against D10 with an $\mathrm{IC}_{50}$ value of $2.3 \mu \mathrm{M}$.

Microglossa pyrifolia, a medicinal plant used against malaria in Ghana, was tested against both PoW and Dd2 strains of P. falciparum by Köhler et al. [36]. Two diterpenes, E-phytol (20) (IC $50: 8.5 \mu \mathrm{M}$ (PoW); $11.5 \mu \mathrm{M}$ (Dd2)), and 6E-geranylgeraniol-19-oic acid (21) (IC 50 : $12.9 \mu \mathrm{M}$ (PoW); $15.6 \mu \mathrm{M}$ (Dd2)) were shown to be the most active compounds in their test system (Figure 6).

A Plasmodium berghei-infected mouse model was used to evaluate the antimalarial activity of the $80 \%$ methanol extract of the roots of the traditionally used antimalarial plant Echinops hoehnelii. The methanol extract could suppress the parasite growth by $68.5 \%$ at a dose of $200 \mathrm{mg} / \mathrm{kg}$. No acute oral toxicity was observed in the animal study, indicating the safety use of the plant extract. Further phytochemical separation of the plant led to the isolation of two acetylenicthiophenes, 5-(penta-1,3-diynyl)-2-(3,4-dihydroxybut-1-ynyl)-thiophene (22) and 5-(penta-1,3-diynyl)-2-(3-chloro-4-acetoxy-but-1-yn)-thiophene (23), which displayed significant growth suppression of the Plasmodium parasite by $50.2 \%$ and $32.7 \%$ at $100 \mathrm{mg} / \mathrm{kg}$, respectively [37] (Figure 6).

\subsection{Buxaceae Family}

Cai et al. identified several new antimalarial compounds from Buxus sempervirens [38], the native and introduced plant species in the United States. The traditionally used plants have received scant attention as potential source materials for drug discovery research as compared to the botanical materials from tropical and semitropical areas of the world. The eight lupane triterpenes (24-31), isolated from the Buxus plant (Figure 7), were evaluated for their activity against multi-drug-resistant malaria parasites (HB3, $\mathrm{IC}_{50} 0.5-3.0 \mu \mathrm{M}$ ) and counterscreened against HeLa cells ( $\mathrm{IC}_{50} 7 \mu \mathrm{M}$ for 24; $>20 \mu \mathrm{M}$ for 25-31). Strikingly, 23-O-(trans)-feruloyl-23-hydroxybetulin (26) displayed antimalarial 
activity at a concentration that was 75-fold more selective to the drug-resistant parasite strain than to HeLa cells.

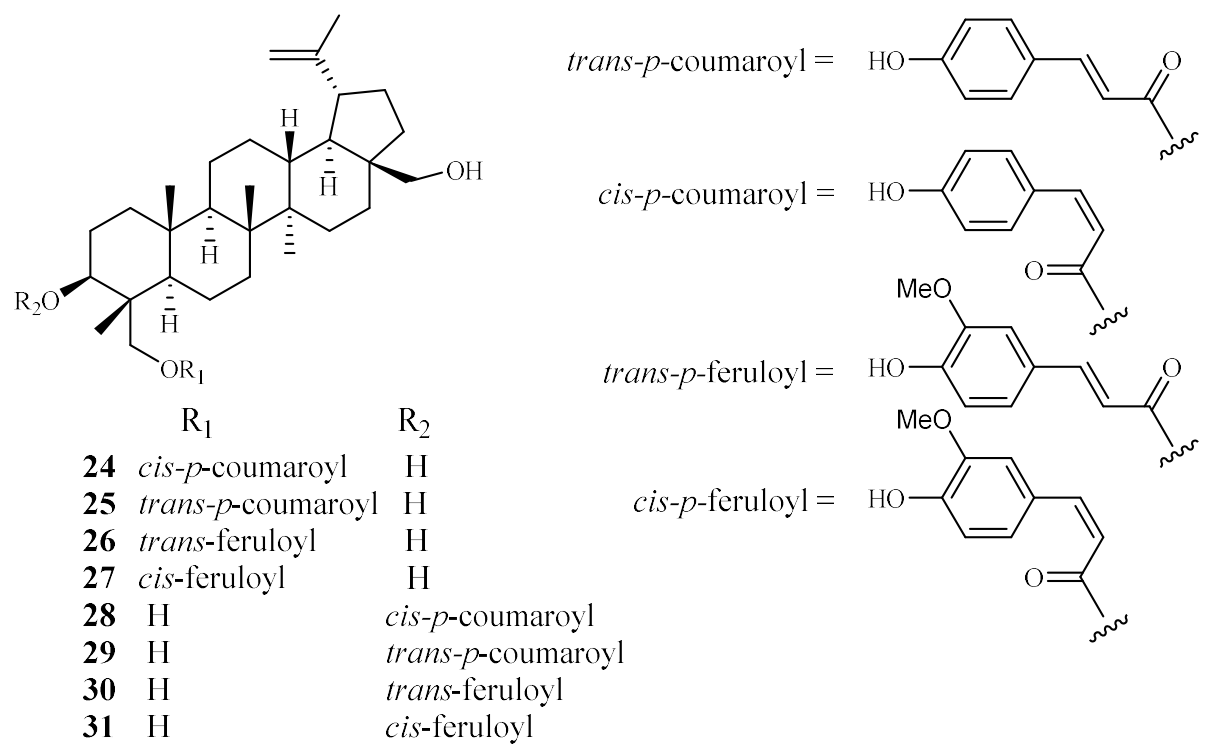

Figure 7. Compounds from a Buxaceae plant.

\subsection{Cecropiaceae-Cucurbitaceae Families}

\subsubsection{Cecropiaceae Family}

Cecropia pachystachya is a medicinal plant, which has been used in Brazil. The ethanol extracts of the different parts of the plants were evaluated for their activity against P. falciparum in vitro and $P$. berghei in vivo [39]. The parasitemia of malaria-infected mice was reduced by $35-66 \%$ with treatment of the ethanol extracts of the wood, root, and leaf materials in comparison with the non-treated control group. The plant root extracts were further analyzed and fractionated to provide subfractions, which were also active in an in vivo study. Two compounds, $\beta$-sitosterol (32) and tormentic acid (33), were identified from the subfractions (Figure 8). Both compounds showed plasmodial inhibitory activity. However, only tormentic acid (33) demonstrated inhibitory activity against $P$. falciparum chloroquine-resistant parasites (W2) ( $\left.\mathrm{IC}_{50} 19.0-25.2 \mu \mathrm{M}\right)$.

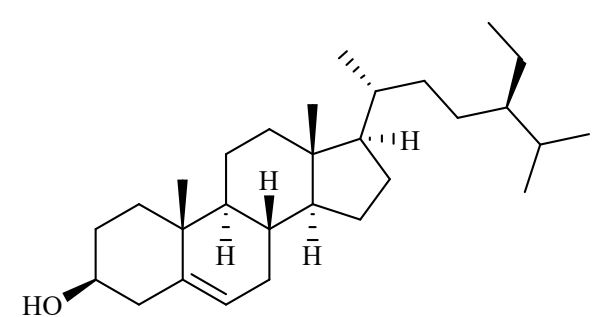

32

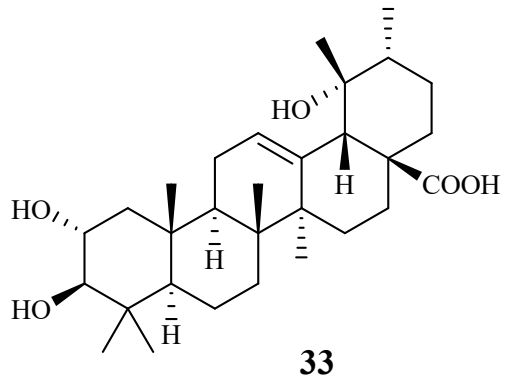

33

Figure 8. Compounds from a Cecropiaceae plant.

\subsubsection{Chloranthaceae Family}

Yue et al. [40] recently reported the isolation of 32 antimalarial lindenane-type sesquiterpenoids (34-65) from several plants in Chloranthaceae family with $\mathrm{IC}_{50}$ values lower than $11.4 \mu \mathrm{M}$ against P. falciparum strain Dd2. The 12 new sesquiterpenoid dimers fortunilides A-L (34-45), along with 7 known isolates (46-51 and 53) were isolated from $C$. fortune. Compounds $\mathbf{5 2}, \mathbf{5 4}, \mathbf{5 8}, \mathbf{5 9}$ and $\mathbf{6 0 - 6 4}$ 
were obtained from C. serratus and C. spicatus, and compounds 55-57 were separated from Sarcandra glabra. Compound 65 was originated from C. multisachys. Among these isolates, fortunilide A (34), sarglabolide J (47) and chlorajaponilide C (52) exhibited low nanomolar activities with $\mathrm{IC}_{50}$ values of 5.2, 7.2 and $1.1 \mathrm{nM}$, respectively, and their selectivity index values toward mammalian cells were greater than 500 (Figure 9).

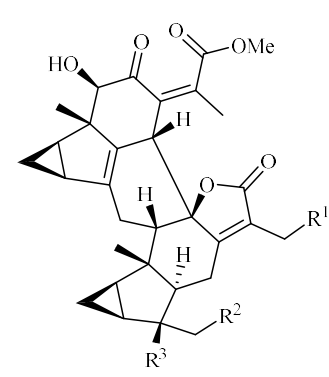

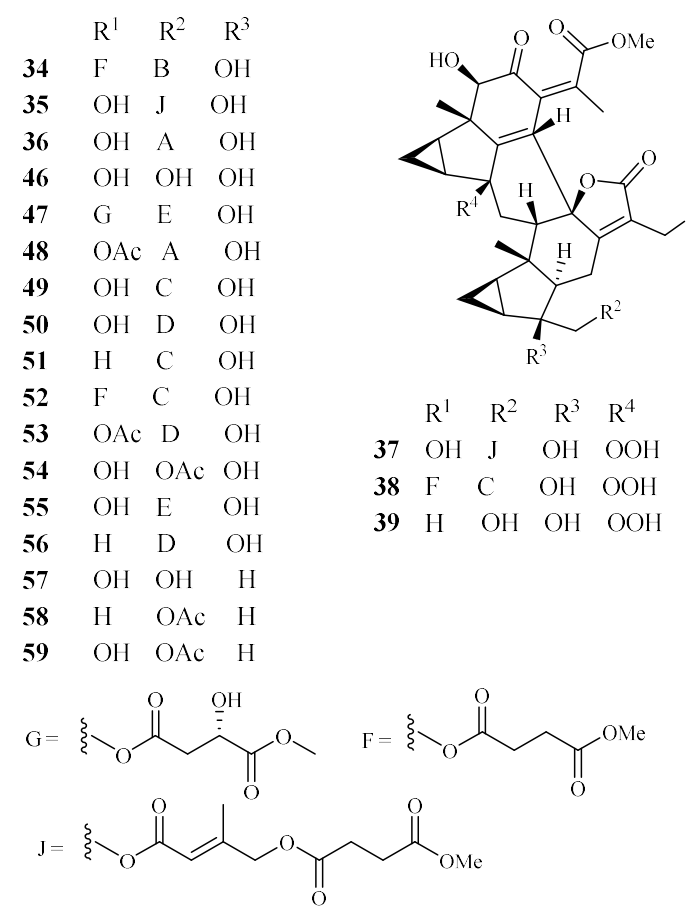

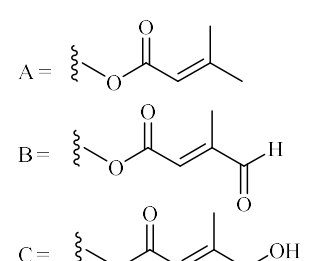

$$
\begin{array}{lllll} 
& \mathrm{R}^{1} & \mathrm{R}^{2} & \mathrm{R}^{3} & \mathrm{R}^{4} \\
37 & \mathrm{OH} & \mathrm{J} & \mathrm{OH} & \mathrm{OOH}
\end{array}
$$<smiles>[CH][CH]O[CH]C=C(C)CO</smiles><smiles>CC=CC(=O)OCCO</smiles><smiles>CC(=CCO)C(=O)OCCO</smiles>

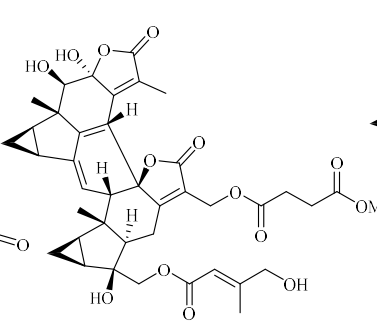

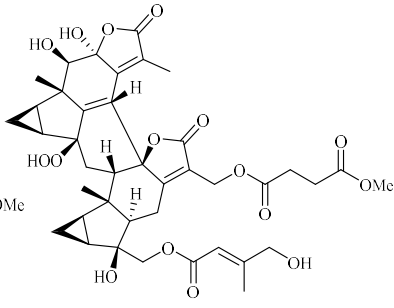

$\mathrm{R}^{1}$ $\mathrm{R}^{2}$

$40 \mathrm{Me}$ COOMe

41

42
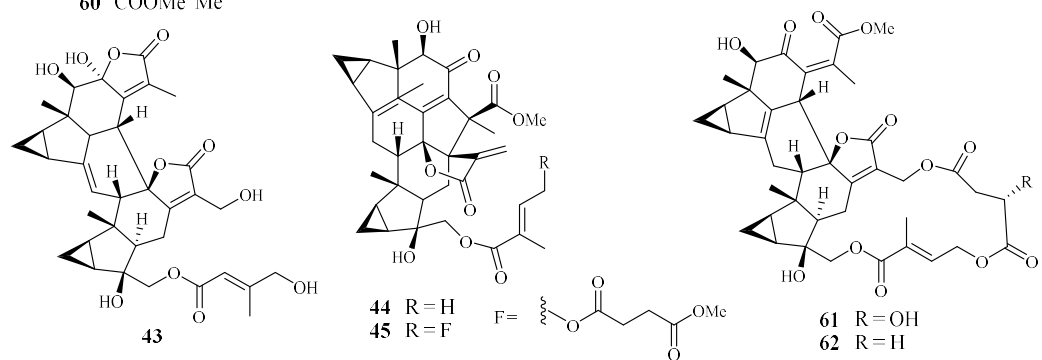

$61 \mathrm{R}=\mathrm{OH}$
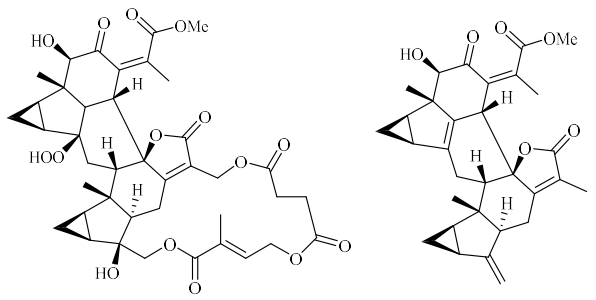

64

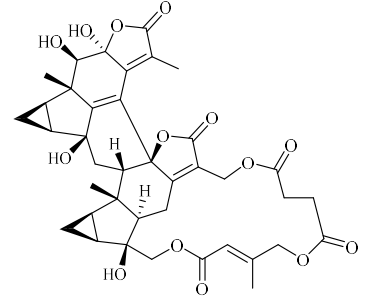

Figure 9. Compounds from Chloranthaceae plants. 


\subsubsection{Chrysobalanaceae Family}

From the Petroleum ether $/ \mathrm{CH}_{2} \mathrm{Cl}_{2}$ extracts of the stems of Parinari capensis, three kaurene diterpene lactones, 10, 13-dihydroxy-9-methyl-15-oxo-20-norkaur-16-en-18-oic acid $\gamma$-lactone (66), 10-hydroxy-13-methoxy-9-methyl-15-oxo-20-norkaur-16-en-18-oic acid $\gamma$-lactone (67) and 10-hydroxy-9-methyl-15-oxo-20-norkaur-16-en-18-oic acid $\gamma$-lactone (68) were isolated (Figure 10) [41]. They possess antimalarial activity against FCR-3 with $\mathrm{IC}_{50}$ values of $1.7,1.9$ and $5.0 \mu \mathrm{M}$, respectively.

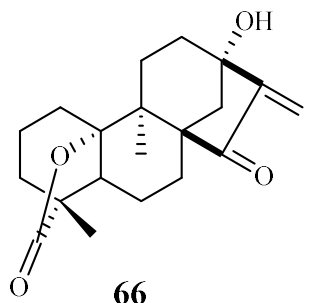

66

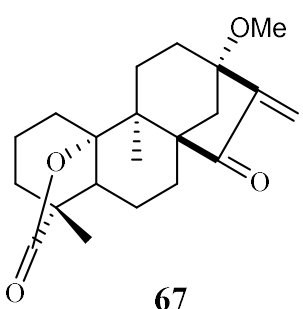

67

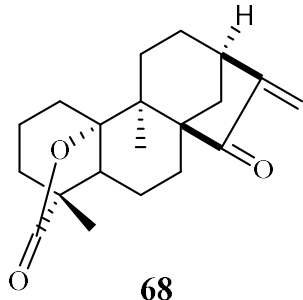

68

Figure 10. Compounds from a Chrysobalanaceae plant.

The three compounds (66-68) also displayed cytotoxicity against Graham cells with ED 50 values in the range of 3.2-9.2 $\mu \mathrm{M}$, which preclude them from further biological investigation. They could, however, be used effectively as lead compounds for drug optimization through synthesis.

\subsubsection{Clusiaceae Family}

Phytochemical separation of the concentrated acetone extract of the dried leaves and branches of Garcinia mckeaniana has led to the identification of three new xanthones, mckeanianones A-C (69-71), and two known ones, bannaxanthones I (73) and E (73) (Figure 11). These compounds all contain two isoprene units. They were evaluated for their activity against the TM4 and $\mathrm{K} 1$ strains of $P$. falciparum with $\mathrm{IC}_{50}$ values in the range of of 6.0-8.5 and 3.6-7.3 $\mu \mathrm{M}$, respectively, and compounds 70, 71 and 73 showed cytotoxicity against Vero cells with the $\mathrm{IC}_{50}$ values in the range of $12.6-29.5 \mu \mathrm{M}$ [42].<smiles>[Y9]c1c(O)c(O)c(Br)c2c(=O)c3c(O)c4c(c(Br)c3oc12)OC(C)(C)C=C4</smiles>

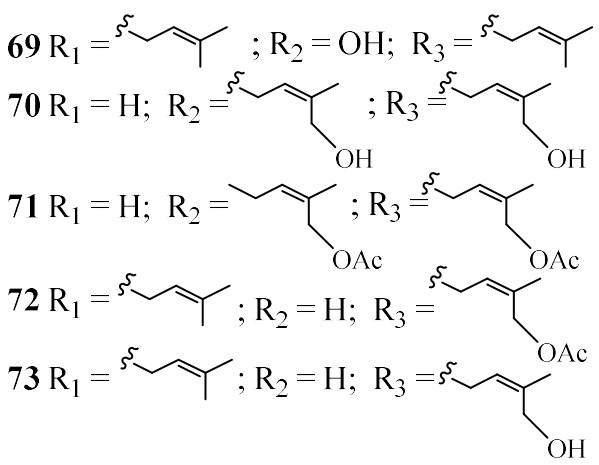

Figure 11. Compounds from a Clusiaceae plant.

\subsubsection{Connaraceae Family}

From the work of He et al. [26], bioassay-guided separation of the chloroform extract of the stems of Rourea minor (Gaertn.) Aubl. led to the identification of three active compounds including two new neolignan glycosides, rourinoside (74) and rouremin (75), and the known 1-(26-hydroxyhexacosanoyl)-glycerol (76) (Figure 12). The three compounds showed weak to moderate in vitro activities against the $\mathrm{D} 6$ and $\mathrm{W} 2$ clones of $P$. falciparum. Compound 74 demonstrated $\mathrm{IC}_{50}$ values at 3.7 (D6) and 2.1 (W2) $\mu \mathrm{M} ; 75$ at $\mathrm{IC}_{50}$ values of 5.1 (D6) and 4.5 (W2) $\mu \mathrm{M}$, and 76 at $\mathrm{IC}_{50}$ values of 9.5 (D6) and 12.7 (W2) $\mu \mathrm{M}$. These compounds exhibited no cytotoxicity against KB cells at 20 $\mu \mathrm{g} / \mathrm{mL}$. 

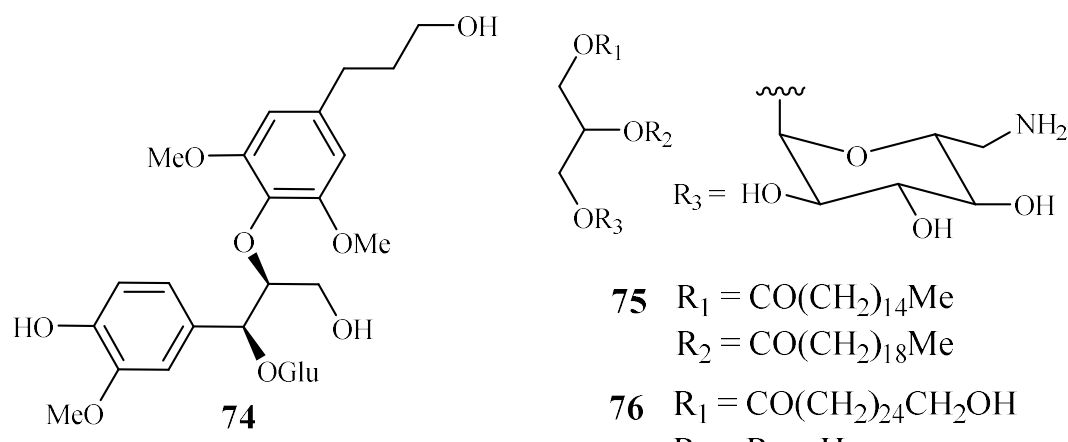

$75 \mathrm{R}_{1}=\mathrm{CO}\left(\mathrm{CH}_{2}\right)_{14} \mathrm{Me}$

$\mathrm{R}_{2}=\mathrm{CO}\left(\mathrm{CH}_{2}\right)_{18} \mathrm{Me}$

$76 \mathrm{R}_{1}=\mathrm{CO}\left(\mathrm{CH}_{2}\right)_{24} \mathrm{CH}_{2} \mathrm{OH}$

$\mathrm{R}_{2}=\mathrm{R}_{3}=\mathrm{H}$

Figure 12. Compounds from a Connaraceae plant.

\subsubsection{Cornaceae Family}

In vitro $\mathrm{IC}_{50}$ values against the $P$. falciparum D10 strain were determined for ergosta-4,6,8,22tetraene-3-one (77) $(61.0 \mu \mathrm{M})$, 3-epideoxyflindissol (78) $(128.0 \mu \mathrm{M})$, 3ß-O-cis-coumaroyl betulinic acid (79) $(10.4 \mu \mathrm{M})$ and 3ß-O-trans-coumaroyl betulinic acid (80) $(15.3 \mu \mathrm{M})$ (Figure 13), which were separated from the leaves of Cornus florida L. by Graziose et al. for the first time [43].

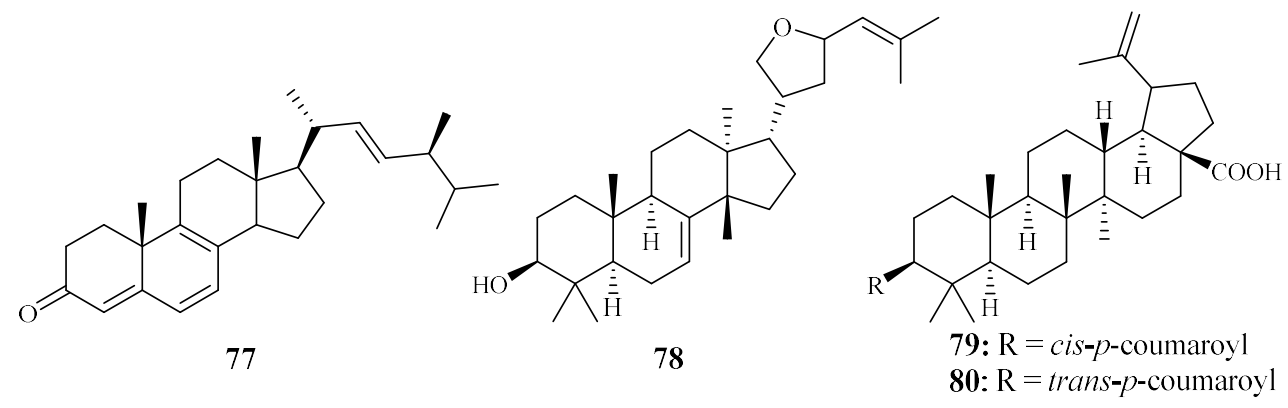

Figure 13. Compounds from a Cornaceae plant.

\subsubsection{Cucurbitaceae Family}

Cogniauxia podolaena Baill. is a folk medicine that has been traditionally used to treat malaria in Congo Brazzaville. Banzouzi et al. [44] identified cucurbitacins B (81) and D (82), and 20-epibryonolic acid (83), the three triterpenes from the stems of this plant (Figure 14). These compounds exhibited inhibitory activity against FcM29 strain with $\mathrm{IC}_{50}$ values of $2.9,7.8$ and $4.4 \mu \mathrm{M}$, respectively. Both cucurbitacins B and D showed a high cytotoxicity with approximately $95 \%$ inhibition against KB cells at $1 \mu \mathrm{g} / \mathrm{mL}$, while 20-epibryonolic acid displayed a better selectivity index ( $20 \%$ inhibition of KB cells at $1 \mu \mathrm{g} / \mathrm{mL}$ ).

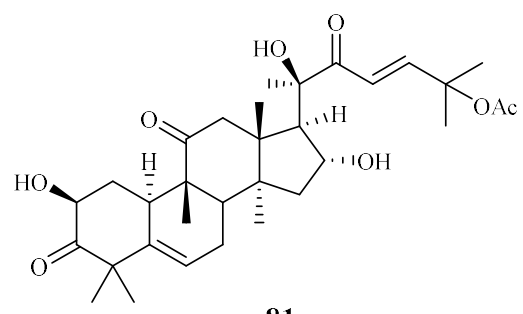

81<smiles>CC(C)(O)/C=C/C(=O)[C@@H](O)[C@H]1[C@@H](O)C[C@]2(C)[C@@H]3CC=C4[C@@H](C[C@H](O)C(=O)C4(C)C)[C@@]3(C)C(=O)C[C@]12C</smiles>

82

Figure 14. Compounds from a Cucurbitaceae plant. 


\subsection{Ebenaceae-Euphorbiaceae Families}

\subsubsection{Ebenaceae Family}

Ma et al. [27] investigated the plant Diospyros quaesita Thw., known as "Muang Kout" in Laos. Of the isolates from the up parts of this plant, betulinic acid 3-caffeate (84) demonstrated antiplasmodial activity against the D6 and W2 clones with $\mathrm{IC}_{50}$ values of 1.40 and $0.98 \mu \mathrm{M}$, respectively (Figure 15). The compound was cytotoxic to KB cells with an $\mathrm{ED}_{50}$ value of $4.0 \mu \mathrm{M}$.

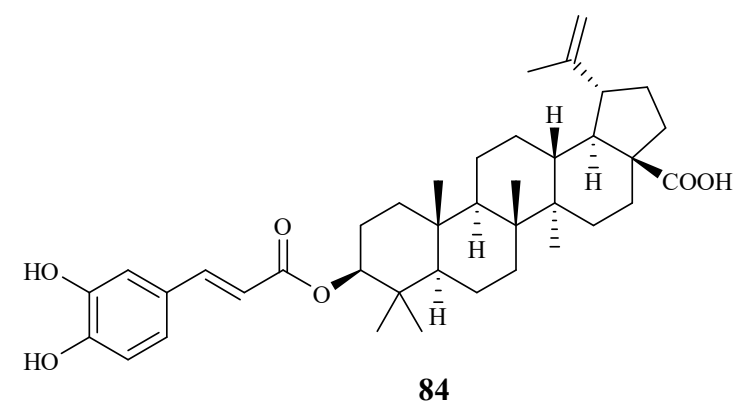

Figure 15. Compounds from an Ebenaceae plant.

\subsubsection{Euphorbiaceae Family}

Through the screening of a natural product-based synthetic compound library, Hadi et al. [45] discovered that jatrophones (the natural products from Jatropha isabelli) possess significant antiplasmodial activity. The jatrophone diterpene derivatives $\mathbf{8 5}$ and $\mathbf{8 6}$ displayed antiplasmodial activities against strains 3D7 and $\mathrm{K} 1$ of $P$. falciparum with $\mathrm{IC}_{50}$ values of 5.7/5.9 and 6.1/5.9 $\mu \mathrm{M}$, respectively (Figure 16). The two compounds showed low cytotoxicities against the human HepG2, RAJI, BJ and HEK293 cells with $\mathrm{EC}_{50}$ values at around $26 \mu \mathrm{M}$.

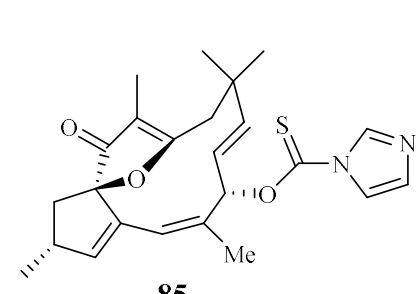

85

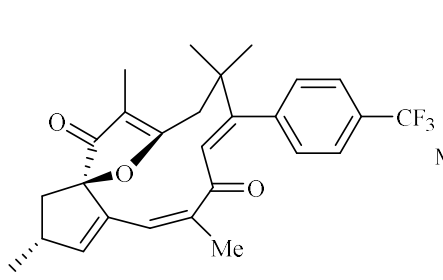

86<smiles>COC1=Cc2c(cc(O)c3cc(C)c(OC)cc23)C(C)(C)C1=O</smiles>

87<smiles>COc1cc2c(cc1C)c(OC)cc1cc(OC)c(=O)c(C)cc12</smiles>

88

Figure 16. Compounds from Euphorbiaceae plants.

Seephonkai et al. [46] studied the Thai traditional medicinal plant Strophioblachia fimbricalyx, and isolated 9-O-demethyltrigonostemone (87) and a new phenanthropolone, 3,6,9trimethoxyphenanthropolone (88), which exhibited antimalarial activity against the multiresistant K1 strain of P. falciparum with $\mathrm{IC}_{50}$ values of 8.7 and $9.9 \mu \mathrm{M}$, respectively (Figure 16).

\subsection{Fabaceae-Fagaceae Families}

\subsubsection{Fabaceae Family}

According to Nigerian ethnobotany, the plant Cajanus cajan L. (Fabaceae) can be used for treatment of malaria. From the methanol extract of the leaves of this plant, 2',6'-dihydroxy-4-methoxy chalcone (89), a cajachalcone, was isolated through bioassay-guided fractionation, which used the parasite lactate dehydrogenase assay by targeting the K1 strain of P. falciparum (Figure 17). The cajachalcone showed an $\mathrm{IC}_{50}$ value of $7.4 \mu \mathrm{M}$ [47]. 


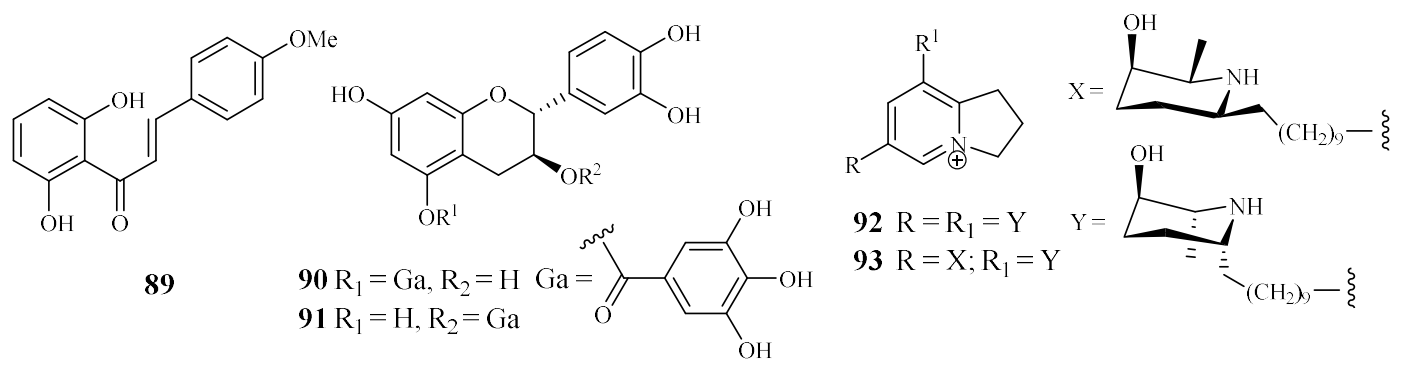

Figure 17. Compounds from Fabaceae plants.

From the work of Ramanandraibe et al. [48], Piptadenia pervillei Vatke was prioritized as an active plant lead identified through a screening program, which was dedicated to discovering antimalarial compounds from the plants in Madagascar. Separation of the EtOAc extract of the leaves of this plant led to the identification of the bioactive compounds (+)-catechin 5-gallate (90) and (+)-catechin 3-gallate (91). The two compounds showed antimalarial activity against $\mathrm{FcB1}$ strain with $\mathrm{IC}_{50}$ values of 1.2 and $1.0 \mu \mathrm{M}$, respectively (Figure 17), and no significant cytotoxicity was observed at $75 \mu \mathrm{M}$ for the two compounds against the human embryonic lung cells MRC-5.

According to the work of Samoylenko et al. [49], prosopilosidine (92) and isoprosopilosidine (93), isolated from the leaves of Prosopis glandulosa var. glandulosa, showed potent antimalarial activity against the D6 and W2 strains of P. falciparum with high selectivity index (SI) values (Figure 17). Compound 92 exhibited IC 50 values of 0.1 (D6) and 0.3 (W2) $\mu \mathrm{M}$, while 93 demonstrated IC $_{50}$ values of 0.1 (D6) and 0.3 (W2) $\mu \mathrm{M}$. Compounds 92 and 93 showed much lower cytotoxicity to KB cells with $\mathrm{ED}_{50}$ values of 20.2 and $18.8 \mu \mathrm{M}$, respectively.

\subsubsection{Fagaceae Family}

Subsequent bioassay-guided fractionation work by Cai et al. [38] yielded four kaempferol 3-O-glucosides (94-97) from Quercus laceyi (Figure 18). The $\mathrm{IC}_{50}$ values for these compounds against multi-drug-resistant malaria parasites $\mathrm{HB} 3$ are $0.6-2.1 \mu \mathrm{M}$, and the $\mathrm{IC}_{50}$ value against HeLa cells was $<3 \mu \mathrm{M}$.

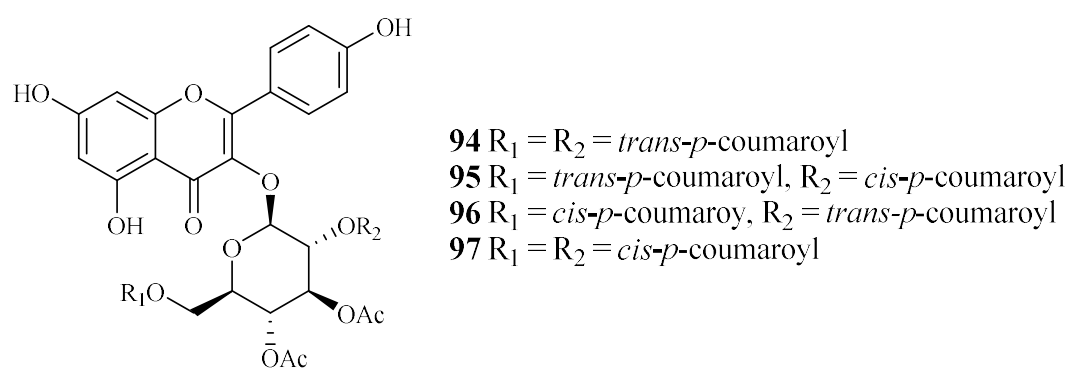

Figure 18. Compounds from a Fagaceae plant.

\subsection{Hypericaceae Family}

Vismia orientalis, a traditional medicine used in Tanzania, was studied by Mbwambo et al. [50]. Vismione $\mathrm{D}$ (98), isolated from the stem barks of this plant, exhibited activity against the K1 strain with an $\mathrm{IC}_{50}$ value of $2.4 \mu \mathrm{M}$ (Figure 18). However, the compound also showed cytotoxicity against human L6 cells with an $\mathrm{IC}_{50}$ value of $10.0 \mu \mathrm{M}$.

Pure isolates from the hexane extract of the stem barks of the African plant Psorospermum glaberrimum were evaluated for their antimalarial activity against the W2 clone of $P$. falciparum by Ndjakou Lenta et al. [51]. The isolates 3-geranyloxyemodin anthrone (99) and acetylvismione 
$\mathrm{D}$ (100) displayed inhibition activity against the W2 strain with $\mathrm{IC}_{50}$ values of 1.7 and $0.1 \mu \mathrm{M}$, respectively (Figure 19).<smiles>CC(C)=CCC/C(C)=C/COc1cc(O)c2c(O)c3c(cc2c1)CC(C)(O)CC3=O</smiles>

98<smiles>CC(C)=CCC/C(C)=C/COc1cc(O)c2c(c1)Cc1cc(C)cc(O)c1C2=O</smiles>

99<smiles>COC1(C)CC(=O)c2c(cc3cc(OC/C=C(\C)CCC=C(C)C)cc(O)c3c2O)C1</smiles>

100

Figure 19. Compounds from Hypericaceae plants.

\subsection{Lamiaceae-Lythraceae Families}

\subsubsection{Lamiaceae Family}

An EtOH extract of the dried root barks of Ocimum sanctum exhibited considerable in vitro antimalarial activity. Bioactivity-directed separation of the $\mathrm{EtOH}$ extract resulted in the isolation of a new antimalarial natural compound (101) (Figure 20). The compound showed comparable activity to the positive controls, chloroquine and amodiaquine, against the P. falciparum 3D7 strains with an $\mathrm{IC}_{50}$ value of $0.1 \mu \mathrm{M}[52]$.<smiles>COc1ccc(-c2cc(=O)c3c(O)c(OC)c(OC)cc3o2)cc1</smiles>

Figure 20. Compounds from Lamiaceae plants.

From the study of Kirmizibekmez et al. [53], luteolin 7-O- $\beta$-D-glucopyranoside (102) and chrysoeriol 7-O- $\beta$-D-glucopyranoside (103), two flavonoid glycosides isolated as the major antimalarial constituents from Phlomis brunneogaleata through an activity-directed separation (Figure 20), showed activity with $\mathrm{IC}_{50}$ values of 5.4 and $12.7 \mu \mathrm{M}$ against the $\mathrm{K} 1$ clones, respectively.

The extracts of 17 Salvia species, which are used as folk medicines in South Africa, were subjected to biological testing by Kamatou et al. [54]. The potential activity of the Salvia plant extracts against the FCR strain of P. falciparum and their cytotoxic effects against MCF-7 cells were investigated. These extracts showed antiplasmodial activity with $\mathrm{IC}_{50}$ values in the range of $3.9-26.0 \mu \mathrm{g} / \mathrm{mL}$. The extracts from S. radula demonstrated the most potent activities. Two compounds, betulafolientriol oxide (104) and salvigenin (105), were subsequently isolated (Figure 20), and they showed antimalarial activity with $\mathrm{IC}_{50}$ values of 10.4 and $75.0 \mu \mathrm{M}$, respectively.

\subsubsection{Loganiaceae Family}

A phytochemical study was carried out for the stem barks of Strychnos icaja for the first time by Tchinda et al. [55], which led to the isolation of the monomers 15-hydroxyvomicine (106) and 
$N$-methyl-sec-iso-pseudostrychnine (107). The isolates were evaluated against the P. falciparum 3D7 strain with $\mathrm{IC}_{50}$ values of 101.0 and $110.6 \mu \mathrm{M}$, respectively (Figure 21).

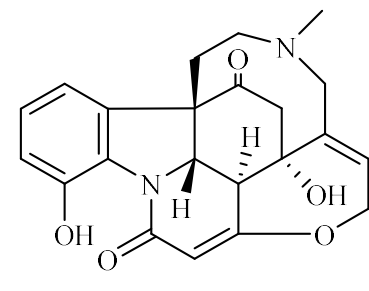

106

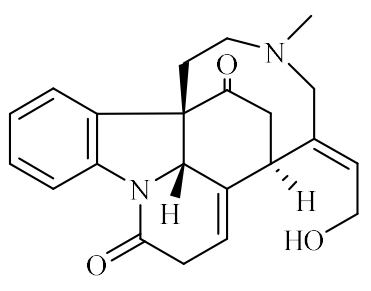

107

Figure 21. Compounds from a Loganiaceae plant.

\subsubsection{Lythraceae Family}

The plants in the genus of Ammannia are frequently used in China and India as folk medicines for treatment of various diseases. Upadhyay et al. [56] investigated the compounds in four species of this genus (Ammannia: A. multiflora, A. verticillata, A. Baccifera and A. coccinea) for their antimalarial activities. Among the isolated compounds, 4-hydroxy- $\alpha$-tetralone (108) and tetralone-4-O- $\beta$-D-glucopyranoside (109) from A. multiflora, and ammaniol (110) from A. baccifera displayed antimalarial activities against the P. falciparum NF-54 strain with $\mathrm{IC}_{50}$ values of $194.0,124.0$ and $88.3 \mu \mathrm{M}$, respectively (Figure 22).

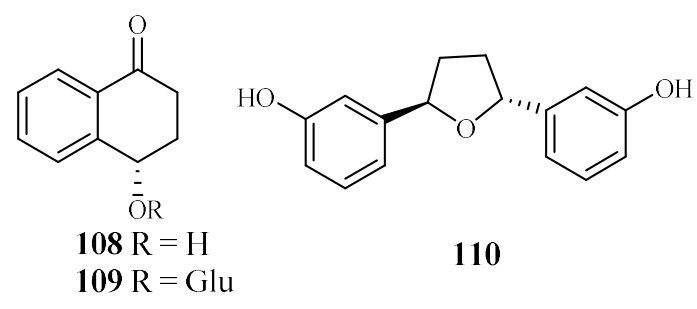

Figure 22. Compounds from Lythraceae plants.

\subsection{Malvaceae-Myristicaceae Families}

\subsubsection{Malvaceae Family}

LC-PDA-MS-SPE-NMR technique was used by Sprogøe et al. in combination with CD to detect $(R)-(-)$-gossypol [(R)-1] (111) in the twigs of Thespesia danis (Figure 23) [57]. (R)-1 demonstrated antimalarial activity with an $\mathrm{IC}_{50}$ value of $4.5 \mu \mathrm{M}$. However, its enantiomer was inactive up to a concentration of $20 \mu \mathrm{M}$.

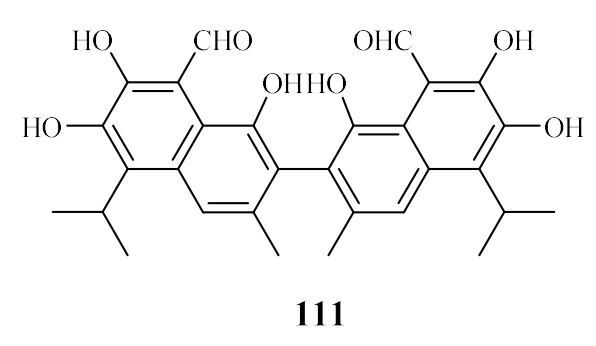

Figure 23. Compounds from a Malvaceae plant.

\subsubsection{Monimiaceae Family}

The compound 1-(4-hydroxybenzyl)-6,7-methylenedioxy-2-methylisoquinolinium trifluoroacetate (112), a new benzylisoquinoline alkaloid isolated by mass-guided separation of the $\mathrm{CH}_{2} \mathrm{Cl}_{2} / \mathrm{MeOH}$ 
extract of Doryphora sassafras (Figure 24) [58]. Compound 112 showed antiplasmodial activity against two different strains (3D7 and Dd2) of P. falciparum with $\mathrm{IC}_{50}$ values of 3.0 and $4.4 \mu \mathrm{M}$, respectively. The compound did not exhibit inhibitory activity against the human embryonic kidney cell line (HEK293) at a concentration of $120 \mu \mathrm{M}$.<smiles>C[n+]1ccc2cc3c(cc2c1Cc1ccc(O)cc1)OCO3</smiles><smiles>[R][R]([H])=[W]</smiles>

Figure 24. Compounds from Monimiaceae plants.

A phytochemical study of the leaves of Glossocalyx brevipes Benth. led to isolation of two new homogentisic acid derivatives of methyl 2-( $1^{\prime} \beta$-geranyl- $5^{\prime} \beta$-hydroxy-2'-oxocyclohex- $3^{\prime}$-enyl) acetate (113) and 2-(1' $\beta$-geranyl-5' $\beta$-hydroxy-2'-oxocyclohex-3'-enyl) acetic acid (114), which displayed antiplasmodial activity against D6/W2 clones with $\mathrm{IC}_{50}$ values of $2.2 / 6.6$ and $4.8 / 8.3 \mu \mathrm{M}$, respectively (Figure 24) [59].

\subsubsection{Moraceae Family}

According to the investigation of Zhang et al. [24], an antimalarial trichothecene compound, verrucarin L acetate (4), was identified from Ficus fistulosa (Figure 2). The antimalarial potency of 4 was equivalent to that of roridin E (3) isolated from Rhaphidophora decursiva, a plant from a different family. However, 4 was observed to be much less cytotoxic to $\mathrm{KB}$ cells $\left(\mathrm{ED}_{50} 0.2 \mu \mathrm{M}\right)$ than 3 .

Bioassay-directed separation of the $\mathrm{MeOH}$ extract of the twigs of Ficus septica afforded three known phenanthroindolizine alkaloids, dehydrotylophorine (115), dehydroantofine (116) and tylophoridicine D (117) by Kubo et al. (Figure 25) [60]. They showed antiplasmodial activity against the P. falciparum 3D7 strain with $\mathrm{IC}_{50}$ values in the range of $0.03-0.4 \mu \mathrm{M}$. Compound 115 also displayed cytotoxicity against the mouse fibroblast cells $\mathrm{L} 929$ with an $\mathrm{IC}_{50}$ value of $8.2 \mu \mathrm{M}$, while the other two compounds showed no toxicity at a concentration of $50 \mu \mathrm{M}$.<smiles></smiles>

115: $R_{1}=O M e, R_{2}=O M e$

116: $R_{1}=O M e, R_{2}=H$

117: $\mathrm{R}_{1}=\mathrm{H}, \mathrm{R}_{2}=\mathrm{OMe}$

Figure 25. Compounds from a Moraceae plant.

\subsubsection{Myristicaceae Family}

Phytochemical investigation of the fruits of Knema glauca by Rangkaew et al. [61] led to the isolation of malabaricone $\mathrm{A}(\mathbf{1 1 8})$ as an active compound against the P. falciparum $\mathrm{K} 1$ strain with an $\mathrm{IC}_{50}$ value of $8.5 \mu \mathrm{M}$ (Figure 26). The compound was cytotoxic towards $\mathrm{KB}$ cell with an $\mathrm{ED}_{50}$ value of $>61$ $\mu \mathrm{M}$. 


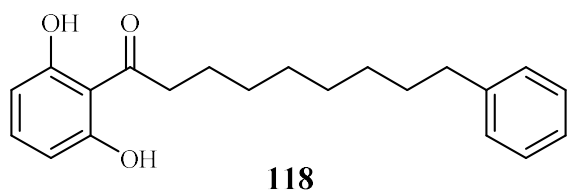

Figure 26. Compound from a Myristicaceae plant.

\subsection{Piperaceae-Platanaceae Families}

\subsubsection{Piperaceae Family}

The Piperaceae family consists of many plants that are used by the native populations in Thailand as traditional medicines for the treatment of various diseases. Sarmentine (119) and 1-piperettyl pyrrolidine (120) were isolated from the fruits of Piper sarmentosum by Rukachaisirikul et al. [62], and they exhibited antiplasmodial activity against the $\mathrm{K} 1$ strain with $\mathrm{IC}_{50}$ values of 85.5 and $21.9 \mu \mathrm{M}$, respectively (Figure 27).

From the whole plant of Piper tricuspe, dictyochromenol (121), 3-farnesyl-p-hydroxy benzoic acid (122) and $2^{\prime} E, 6^{\prime} E$-2-farnesyl hydroquinone (123) were isolated by Saez Vega et al. [63] (Figure 27). The compounds are active against several strains of $P$. falciparum with $\mathrm{IC}_{50}$ values ranging from 1.4 to $29.8 \mu \mathrm{M}$. Cytotoxic effects were also observed for the compounds with $\mathrm{EC}_{50}$ values in the range of $1.1-41.0 \mu \mathrm{M}$. The results suggest that the antimalarial activity of the compounds was most probably the direct result of their cytotoxicity.

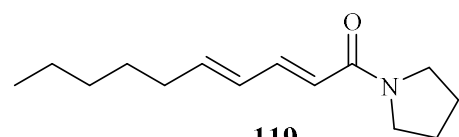

119<smiles>O=C(/C=C/C=C/C=C/c1ccc2c(c1)OCO2)N1CCCC1</smiles>

120<smiles>CC(C)=CCC/C(C)=C/CCC1(C)C=Cc2cc(O)ccc2O1</smiles>

121<smiles>CC(C)=CCC/C(C)=C/CC/C(C)=C/Cc1cc(C(=O)O)ccc1O</smiles><smiles>CC(C)=CCCC(C)=CCCC(C)=CCc1cc(O)ccc1O</smiles>

Figure 27. Compounds from Piperaceae plants.

\subsubsection{Platanaceae Family}

Bioactivity-guided fractionation of Platanus occidentalis by Cai et al. [38] yielded four kaempferol 3-O-rhamnosides (124-127) (Figure 28). The $\mathrm{IC}_{50}$ values for these compounds against multi-drugresistant malaria parasites $\mathrm{HB} 3$ ranged from 0.5 to $1.8 \mu \mathrm{M}$. The $\mathrm{IC}_{50}$ values against HeLa cells were in the range of $9.3-20.0 \mu \mathrm{M}$.

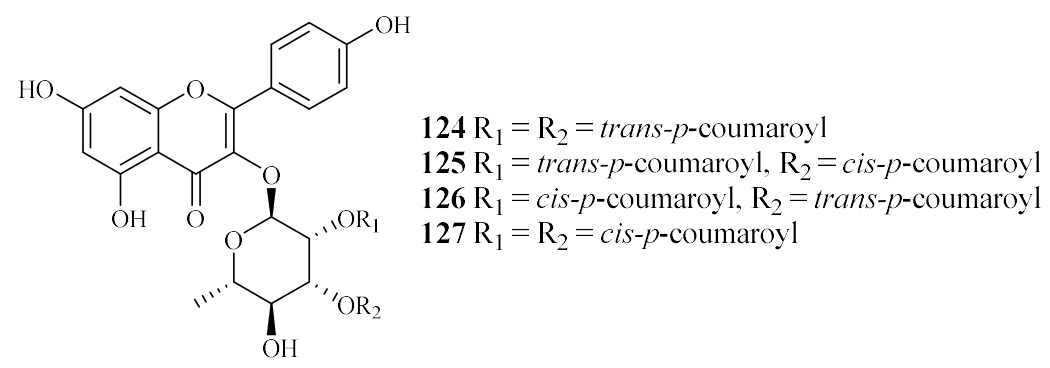

Figure 28. Compounds from a Platanaceae plant. 


\subsection{Rubiaceae-Rutaceae Families}

\subsubsection{Rubiaceae Family}

Naucleaorine (128), epimethoxynaucleaorine (129), 3 3,23-dihydroxyurs-12-en-28-oic acid (130) and oleanolic acid (131) were identified from the stems of Nauclea orientalis by He et al. [28] (Figure 29). The compounds showed antiplasmodial activities against the P. falciparum D6 $\left({ }^{*}\right) / \mathrm{W} 2\left(^{* *}\right)$ strains with the $\mathrm{IC}_{50}$ values shown as below: compound $128\left(\mathrm{IC}_{50} 6.9^{*} / 6.0^{* *} \mu \mathrm{M}\right) ; 129\left(\mathrm{IC}_{50} 12.4^{*} / 13.2^{* *} \mu \mathrm{M}\right) ; 130$ $\left(\mathrm{IC}_{50} 9.7^{*} / 12.7^{* *} \mu \mathrm{M}\right)$ and $\mathbf{1 3 1}\left(\mathrm{IC}_{50} 4.6^{*} / 5.1^{* *} \mu \mathrm{M}\right)$. Compounds 128-131 displayed cytotoxicity against $\mathrm{KB}$ cells with $\mathrm{ED}_{50}$ values of $38.0,>37.9,>42.2$ and $46.0 \mu \mathrm{M}$, respectively.<smiles>C=CC1c2cc3n(c(=O)c2[C@@H](O)OC1OC)CCc1c-3[nH]c2ccccc12</smiles>

128<smiles>C=CC1c2cc3n(c(=O)c2CCc2c-3[nH]c3ccccc23)C(C)[C@H](OC)OC1C</smiles>

139

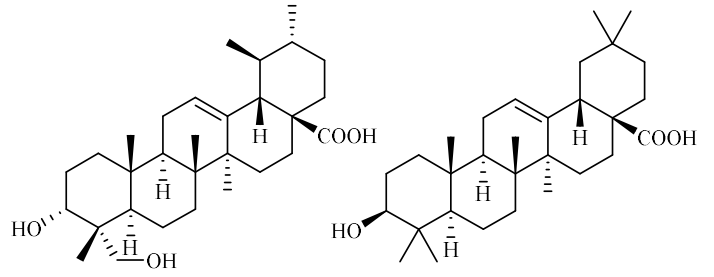

130
131

Figure 29. Compounds from a Rubiaceae plant.

\subsubsection{Rutaceae Family}

Based on an ethnomedicinal survey of the plants in Uganda, Citropsis articulata was selected for phytochemical study to investigate its antimalarial constituents [64]. From the ethyl acetate extract of the root barks of this plant, two known alkaloids, 5-hydroxynoracronycine (132) and 1,5-dihydroxy-2,3-dimethoxy-10-methyl-9-acridone (133), were identified as the best growth inhibitors of $P$. falciparum with $\mathrm{IC}_{50}$ values of 2.8 and $10.0 \mu \mathrm{M}$, respectively. The compounds were cytotoxic towards Vero cells at $\mathrm{EC}_{50}$ values of 28.8 and 101.0, respectively.<smiles>Cn1c2c(cc(O)c3c(=O)c4cccc(O)c4c1=3)OC(C)(C)C=C2</smiles>

132<smiles>COc1cc2c(c(O)c1OC)c(=O)c1cccc(O)c1n2C</smiles>

133<smiles>COc1c2c(c3ccc(=O)oc3c1/C=C/C(C)(C)O)OC(C)(C)C=C2</smiles>

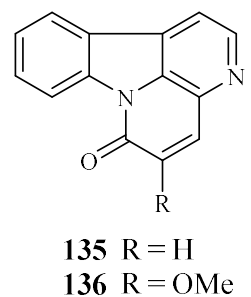

Figure 30. Compounds from Rutaceae plants.

The roots and stem barks of Zanthoxylum chiloperone have been used as a folk medicine for the treatment of malaria and for its emmenagogue and antirheumatic properties. The pyranocoumarin trans-avicennol (134) and two canthinone alkaloids, canthin-6-one (135) and 5-methoxycanthin-6-one (136), were identified from the stem barks of this plant by Cebrián-Torrejón et al. [65] (Figure 30). These compounds possessed antiplasmodial $\mathrm{IC}_{50}$ values against chloroquine/mefloquine resistant and sensitive strains of $P$. falciparum (F32, K1, PFB and FcB1 cells) in the range of 1.4-41.6 $\mu \mathrm{M}$. Compounds 134 and 135 were cytotoxic towards MCR5 cells with $\mathrm{EC}_{50}$ values of 12.8 and $42.7 \mu \mathrm{M}$, respectively.

\subsection{Simaroubaceae Family}

Kuo et al. [66] found that among the isolates from the roots of Eurycoma longifolia, eurycomanone (137) and pasakbumin B (138) [67] displayed potent antimalarial activity against the P. falciparum W2/D6 strains with $\mathrm{IC}_{50}$ values of $0.04 / 0.06$ and $0.05 / 0.08 \mu \mathrm{M}$, respectively (Figure 31). The compounds also exhibited strong cytotoxicity toward human breast cancer (MCF-7) and lung cancer (A549) cells at low concentrations. 
De Andrade-Neto et al. [68] studied a number of Simaroubaceous plants, resulting in the isolation of the following compounds: the quassinoid neosergeolide (139) from the roots and stems of Picrolemma spruce (Figure 31); the indole alkaloids ellipticine (140) and aspidocarpine (141) from the barks of Aspidosperma vargasii and A. desmanthum (Apocynaceae), respectively; and 4-nerolidylcatechol (142) from the roots of Pothomorphe peltata (Piperaceae). Compounds 139-141 presented significant inhibitory activity against the multi-drug resistant $\mathrm{K} 1$ strain with $\mathrm{IC}_{50}$ values of $0.002,0.07,0.02$ and $0.7 \mu \mathrm{M}$, respectively, and these compoundsdisplayed antimalarial potency greater than those of quinine and chloroquine.

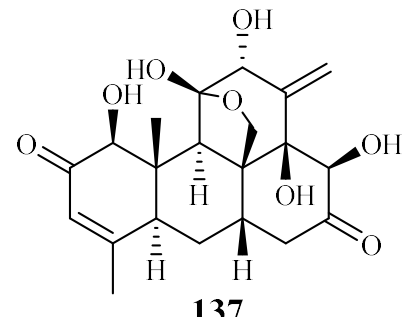

137<smiles>Cc1c2cnccc2c(C)c2c1[nH]c1ccccc12</smiles>

140

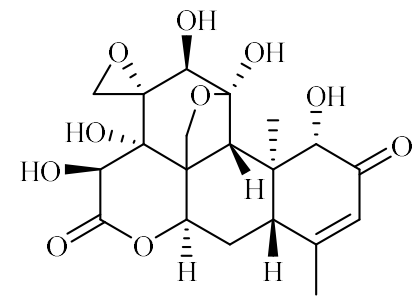

138

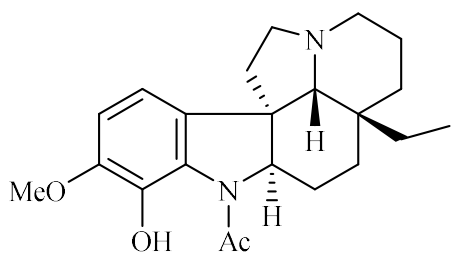

141

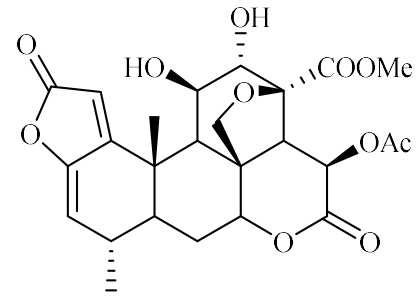

139<smiles>C=CC(C)(CC/C=C(\C)CCC=C(C)C)c1ccc(O)c(O)c1</smiles>

Figure 31. Compounds from Simaroubaceae plants.

\subsection{Theaceae-Tiliaceae Families}

\subsubsection{Theaceae Family}

Gallocatecin (143) is a flavonoid contained in the tea leaf extract of Camellia sinensis (Figure 32). Based on molecular docking studies, Tegar et al. [69] found that gallocatecin has stronger antimalarial potency than mefloquine (144), a synthetic drug with antimalarial activity.<smiles>Cc1c(O)cc(C2Oc3cc(O)cc(O)c3CC2O)cc1O</smiles><smiles>OC(c1cc(C(F)(F)F)nc2c(C(F)(F)F)cccc12)C1CCCCN1</smiles>

Figure 32. Compound from Theaceae plants.

\subsubsection{Tiliaceae Family}

According to the study of Ma et al. [29], five isolates from the leaves, stems and twigs of Grewia

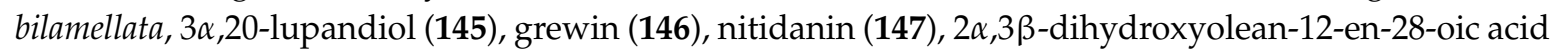
(148) and 2,6-dimethoxy-1-acetonylquinol (149), displayed antimalarial activity against the P. falciparum $\mathrm{D} 6$ and $\mathrm{W} 2$ clones with $\mathrm{IC}_{50}$ values in the range of 5.5-42.2 $\mu \mathrm{M}$ (Figure 33). These compounds showed no cytotoxicity towards KB carcinoma cell line at a concentration of $50 \mu \mathrm{M}$. 


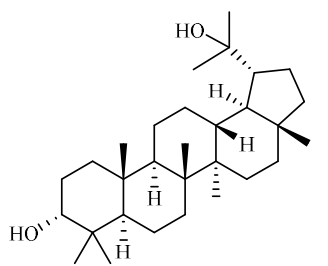

145

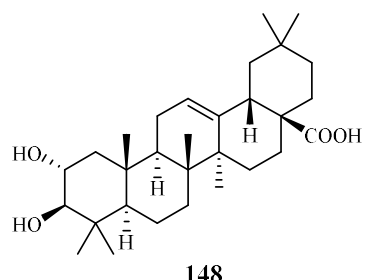<smiles>COc1cc([C@H]2Oc3cc4ccc(=O)oc4cc3O[C@@H]2CO)cc(O)c1O</smiles>

146<smiles>COc1cc(C2Oc3cc(/C=C/CO)cc(OC)c3OC2CO)cc(OC)c1O</smiles>

147

Figure 33. Compounds from a Tiliaceae plant.

\subsection{Verbenaceae Family}

Chromatographic separation of the ethyl acetate extract of the aerial parts of Lippia javanica yielded a new antimalarial $\alpha$-pyrone, lippialactone (150) (Figure 34). This compound is active against the D10 strain with an $\mathrm{IC}_{50}$ value of $23.8 \mu \mathrm{M}$. Compound 119 is also mildly cytotoxic [70].

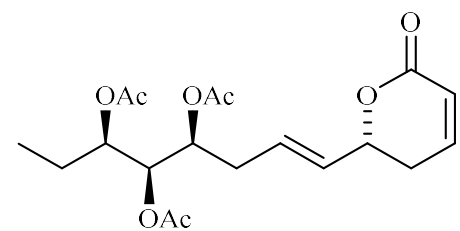

150

Figure 34. Compound from a Verbenaceae plant.

\section{Marine Plant-Derived Antimalarial Compounds}

Marine organisms offer unique opportunity to discover lead compounds for the treatments of malaria.

Separation of the extracts of Fijian red alga Callophycus serratus by Lane et al. led to the isolation of bromophycolides J-Q (151-158) [94] (Figure 35), the macrolide diterpene-benzoate derivatives represented as two novel carbon skeletons. These diterpenes, together with the previously reported ten bromophycolides, bromophycolides A-I (159-167) and debromophycolide A (168) from this alga (Figure 36) [95], were evaluated for their antimalarial activity against $P$. falciparum. The $\mathrm{IC}_{50}$ values of bromophycolides A, D, E, H and M (159, 162, 163, 164 and 154) were observed to be $0.9,0.3,0.8,0.9$ and $0.5 \mu \mathrm{M}$, respectively. Some of these compounds also exhibited strong cytotoxicity toward DU4475, a human breast cancer cell line. The $\mathrm{ED}_{50}$ values of bromophycolides $\mathrm{N}$ and $\mathrm{Q}$ (155 and 158) were 1.5 and $2.0 \mu \mathrm{M}$, respectively. 

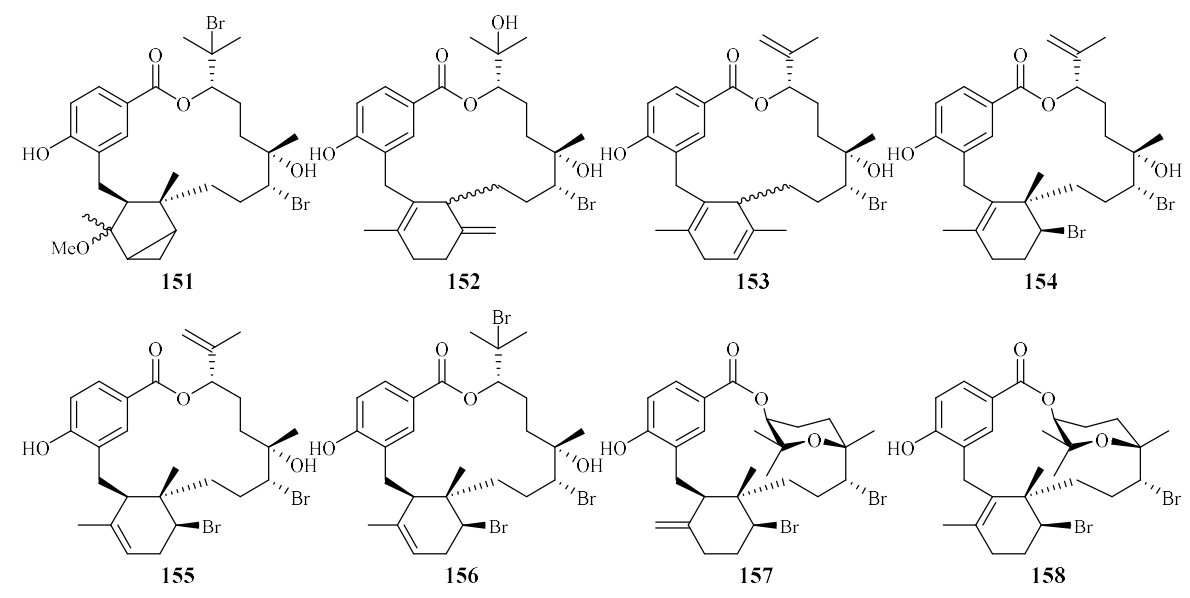

Figure 35. Compounds (151-158) from the red alga Callophycus serratus.

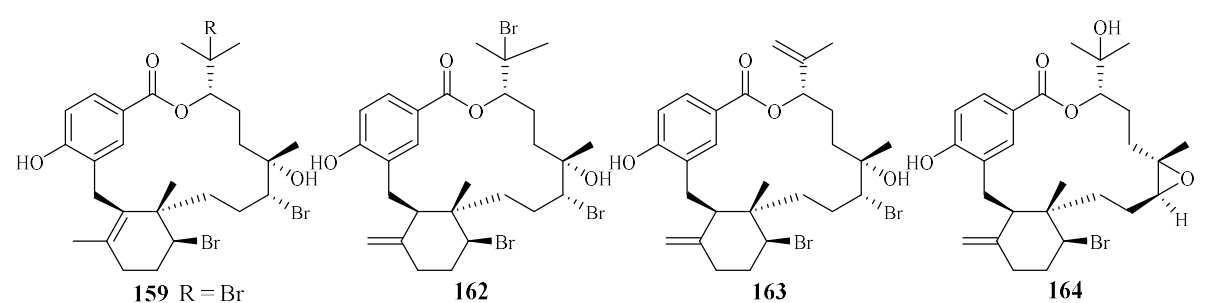

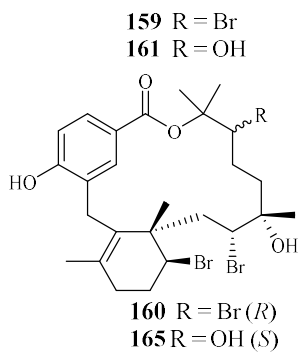

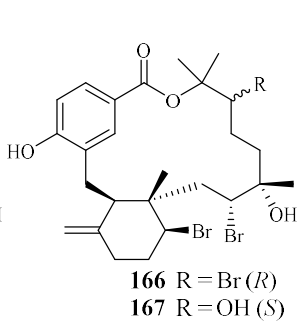<smiles>CC[C@@H]1O[C@]1(C)CCC(OC(=O)c1ccc(O)c(CC2=C(C)CCC2)c1)C(C)(C)O</smiles>

168

Figure 36. Compounds (159-168) from the red alga Callophycus serratus.<smiles>CCCCC1(C)C(C)CCCC1(C)CC/C=C(\C)CCC1(C)CC[C@@H]([C@@H](C)C(C)=O)OO1</smiles><smiles>CC(=O)[C@H](C)[C@H]1C[C@]2(C)C[C@@](CC/C=C(\C)CCC3(C)C(C)CCCC3(C)O)(C1)OO2</smiles>

170<smiles>C=C1CCCC(C)(C)C1(O)CC/C(C)=C/CC[C@@]1(C)C[C@@H]2OO[C@H]([C@@H](C)C(C)=O)C21C</smiles>

171<smiles>C=C1CCCC(C)(C)C1(O)CC/C(C)=C/CC[C@@]1(C)C[C@@H]2OO[C@H]([C@@H](C)C(=O)O)C21C</smiles><smiles>CC1=C2CC/C(C)=C/CC[C@@](C)(C[C@@H]3C[C@@H]([C@@H](C)C(=O)O)OO3)C(CCCC2(C)C)C1</smiles>

173<smiles>CC(=O)C(C)[C@H]1CC[C@](C)(CCC2=C(C)C(=O)CCC2(C)C)OO[C@@H]1CCC1(C)CC[C@@H]([C@H](C)C(=O)O)OO1</smiles>

Figure 37. Compounds from the sponge Diacarnus megaspinorhabdosa.

From the sponge Diacarnus megaspinorhabdosa collected in Xisha Islands, four new norterpene cyclic peroxides, diacarnuperoxides $M$ (169) and $N(\mathbf{1 7 0}),(+)-2,3$, 6-epihurghaperoxide (171) and (+)-2,3,6-epihurghaperoxide acid (172), together with the known norterpene cyclic peroxides, 
(-)-muqubilin A (173), nuapapuin A (174) and diacarperoxide A (175) were isolated by Yang et al. [96] (Figure 37). They exhibited inhibitory activity against $\mathrm{W} 2$ clones of the malaria parasite $P$. falciparum with $\mathrm{IC}_{50}$ values of $4.2,3.0,1.6,4.9,5.6,5.5$ and $1.6 \mu \mathrm{M}$, respectively.

\section{Ethnologic Antimalarial Compounds}

At present, more than $80 \%$ of the world's population relies on ethnopharmacologic healing modalities and plants for their primary health care and wellness [97]. In Africa and many other developing countries, ethnomedicines are often regarded as their primary choice to treat diseases as they are obtained most affordable and accessible from locally available plants or other natural sources [78]. Plants are the major resource for the treatment of malaria infections in sub-Saharan Africa, where health care facilities are limited [98]. Ethnomedicinal plants have played a pivotal role in the treatment of malarial for centuries [71,99].

Early writing of over 6000 years ago in Egypt and China, and those of the Vedic civilisation dated 1600 B.C. in India, indicate that malaria has afflicted humans since antiquity, and there is ample evidence that antimalarial traditional medicaments have been used in virtually all cultures as the mainstay for the treatment of this disease. In the 5th century B.C., Hippocrates rejected superstition as a cause for the fevers that afflicted ancient Greeks. He instead recognized the seasonality of fevers and described the early clinical manifestations and complication of malaria [71].

The widely used antimalarial drug, artemisinin, was isolated from the traditional Chinese herb Artemisia annua L. (Qinghao) [11], which has been used in China as an ethnomedicine for close to 2000 years. The treatment of malaria with Qinghao was first recorded in "Zhouhou Bei Ji Fang", the handbook of prescriptions for emergencies in 243 A.D. [71,77].

The use of ethnomedicine such as herbs for the treatment of malaria varies by region, environment and population subgroups. It may be more preferred in some areas than in others. In order to explore the ethnologic basis of these antimalarial plants, several hundred species from 50 families are presently reviewed and listed in Table 3. These plants were collected from 13 countries and areas, exemplified by Madagascar, Nigeria, South Africa and India. The antimalarial activity and toxicity of these plants are also presented in the table $[7,64,72-76,79-92,100]$.

\section{Conclusions}

It is imperative that the search for new antimalarial agents continues at an unabated pace in order to meet the challenges posed by the development of antimalarial drug resistance. During the last decade, numerous antimalarial compounds have been isolated from plants, and many of these compounds exhibit significant activity against $P$. falciparum in vitro. It is, therefore, evident that plant secondary metabolites continue to play an important role in pre-clinical antimalarial drug discovery.

We present in this comprehensive review, the structures of 175 plant-derived antiplasmodial compounds that have been published during the period of 2001-2017. The relevant plants are organized according to the geographical origins of their corresponding plant families.

Among the 175 plant-derived antiplasmodial compounds, several classes of compounds that showed nanomolar range of activity can be regarded as lead compounds to further explore their antimalarial potential. The trichothecene roridin E (3) from Rhaphidophora decursiva (Araceae family) showed potent inhibitory effects against the parasite growth with $\mathrm{IC}_{50}$ values in the sub-nano molar range $\left(\mathrm{IC}_{50}: 0.4 \mathrm{nM}(\mathrm{D} 6), 1 \mathrm{nM}(\mathrm{W} 2)\right)$ with high cytotoxicity against $\mathrm{KB}$ cells $\left(\mathrm{ED}_{50}: 0.4 \mathrm{nM}\right)$. However, its closely related structural analog, verrucarin L acetate (4), identified from Ficus fistulosa (Moraceae family), displayed much lower cytotoxicity to $K B$ cells $\left(E_{50} 200 \mathrm{nM}\right)$ while retaining the same level of the antiplasmodial activity as 3. Identified from the plant (Ficus septica) in the same genus as that of 4 , the phenanthroindolizine alkaloids dehydroantofine (116) and tylophoridicine $\mathrm{D}$ (117) demonstrated potent antiplasmodial activity against the $P$. falciparum $3 \mathrm{D} 7$ strain with $\mathrm{IC}_{50}$ values of 30 and $60 \mathrm{nM}$, respectively, and the compounds showed no toxicity at a concentration of $50 \mu \mathrm{M}$. A recent study found that the lindenane-type sesquiterpenoids fortunilide A (34), sarglabolide J (47) and chlorajaponilide C 
(52) from the plant in Chloranthaceae family displayed potent antiplasmodial activity against Dd2 strain of with $\mathrm{IC}_{50}$ values of 5.2, 7.2 and $1.1 \mathrm{nM}$, respectively, and these compounds also showed low cytotoxicity to the mammalian cells WI-38 with $\mathrm{IC}_{50}$ values of $8.8,4.0$ and $5.4 \mu \mathrm{M}$, respectivley. More prominently, fortunilide E (38) containing a peroxide group showed antiplasmodial activity of $43 \mathrm{nM}$ with no cytotoxicity at $100 \mu \mathrm{M}$.

This review also describes 25 antimalarial compounds that were reported from marine plants during the time period covered. In addition, we included ethnologic information on antimalarial plants from 50 families that are used as folk medicines for the treatment of malaria. Taken together, all the information presented attests to the fact that the phytochemical investigation of terrestrial plants coupled with the biological validation of ethnomedicines constitute proven strategies for the discovery of potential lead compounds for antimalarial drug development.

Author Contributions: All authors contributed to surveying the literature, preparation and editing of the manuscript.

Acknowledgments: This work was supported by the Research Grants Council of the Hong Kong Special Administrative Region, China (Project No. HKBU 12103014) and the Hong Kong Baptist University (HKBU) Interdisciplinary Research Matching Scheme (RC-IRMS/15-16/02).

Conflicts of Interest: The authors declare no conflict of interest.

\section{References}

1. World Health Organization. World Malaria Report 2017; WHO Press: Geneva, Switzerland, 2017.

2. Mueller, I.; Zimmerman, P.A.; Reeder, J.C. Plasmodium malariae and Plasmodium ovale - The "bashful" malaria parasites. Trends Parasitol. 2007, 23, 278-283. [CrossRef] [PubMed]

3. Collins, W.E. Plasmodium knowlesi: A malaria parasite of monkeys and humans. Annu. Rev. Entomol. 2012, 57, 107-121. [CrossRef] [PubMed]

4. Kajfasz, P. Malaria prevention. Int. Marit. Health 2009, 60, 67-70. [PubMed]

5. Beare, N.A.; Taylor, T.E.; Harding, S.P.; Lewallen, S.; Molyneux, M.E. Malarial retinopathy: A newly established diagnostic sign in severe malaria. Am. J. Trop. Med. Hyg. 2006, 75, 790-797. [PubMed]

6. Mehlhorn, H. Encyclopedia of Parasitology, 3rd ed.; Springer: New York, NY, USA, 2008.

7. Dolabela, M.F.; Oliveira, S.G.; Peres, J.M.; Nascimento, J.M.; Póvoa, M.M.; Oliveira, A.B. In vitro antimalarial activity of six Aspidosperma species from the state of Minas Gerais (Brazil). An. Acad. Bras. Ciênc. 2012, 84, 899-910. [CrossRef] [PubMed]

8. Boulos, M.; Dutra, A.P.; DiSanti, S.M.; Shiroma, M.; Amato, N.V. The clinical evaluation of quinine for the treatment of Plasmodium falciparum malaria. Rev. Soc. Bras. Med. Trop. 1997, 30, 211-213. [CrossRef] [PubMed]

9. Guerin, P.J.; Olliaro, P.; Nosten, F.; Druilhe, P.; Laxminarayan, R.; Binka, F.; Kilama, W.L.; Ford, N.; White, N.J. Malaria: Current status of control, diagnosis, treatment, and a proposed agenda for research and development. Lancet Infect. Dis. 2002, 2, 564-573. [CrossRef]

10. Fidock, D.A.; Rosenthal, P.J.; Croft, L.; Brun, R.; Nwaka, S. Antimalarial drug discovery: Efficacy models for compound screening. Nat. Rev. Drug. Discov. 2004, 3, 509-520. [CrossRef] [PubMed]

11. Tu, Y. The discovery of artemisinin (qinghaosu) and gifts from Chinese medicine. Nat. Med. 2011, 17, 1217-1220. [CrossRef] [PubMed]

12. Klayman, D. Qinghaosu (artemisinin): An antimalarial drug from China. Science 1985, 228, 1049. [CrossRef] [PubMed]

13. Sriram, D.; Rao, V.S.; Chandrasekhara, K.V.; Yogeeswari, P. Progress in the research of artemisinin and its analogues as antimalarials: An update. Nat. Prod. Res. 2004, 18, 503-527. [CrossRef] [PubMed]

14. Zhang, Y.K.; Ge, M.; Plattner, J.J. Recent Progress in the synthesis of antimalarial agents. Org. Prep. Proced. Int. 2012, 44, 340-374. [CrossRef]

15. Kappe, S.H.; Vaughan, A.M.; Boddey, J.A.; Cowman, A.F. That was then but this is now: Malaria research in the time of an eradication agenda. Science 2010, 328, 862-866. [CrossRef] [PubMed]

16. Ginsburg, H.; Atamna, H. The redox status of malaria-infected erythrocytes: An overview with an emphasis on unresolved problems. Parasite 1994, 1, 5-13. [CrossRef] [PubMed] 
17. Zani, B.; Gathu, M.; Donegan, S.; Olliaro, P.L.; Sinclair, D. Dihydroartemisinin-piperaquine for treating uncomplicated Plasmodium falciparum malaria. Cochrane Database Syst. Rev. 2014, 1, 1-160. [CrossRef] [PubMed]

18. Lisewski, A.M.; Quiros, J.P.; Ng, C.L.; Adikesavan, A.K.; Miura, K.; Putluri, N.; Eastman, R.T.; Scanfeld, D.; Regenbogen, S.J.; Altenhofen, L.; et al. Supergenomic network compression and the discovery of EXP1 as a glutathione transferase inhibited by artesunate. Cell 2014, 158, 916-928. [CrossRef] [PubMed]

19. Schlitzer, M. Antimalarial drugs-What is in use and what is in the pipeline. Arch. Pharm. Chem. Life Sci. 2008, 341, 149-163. [CrossRef] [PubMed]

20. Newman, D.J.; Cragg, G.M. Natural products as sources of new drugs from 1981 to 2014. J. Nat. Prod. 2016, 79, 629-661. [CrossRef] [PubMed]

21. Zhang, H.J.; Li, W.F.; Fong, H.H.S.; Soejarto, D.D. Discovery of bioactive compounds by UIC-ICBG drug discovery program in the 18 years since 1998. Molecules 2016, 21, 1448. [CrossRef] [PubMed]

22. Zhang, H.J.; Tamez, P.A.; Vu, D.H.; Ghee, T.T.; Nguyen, V.H.; Le, T.X.; Le, M.H.; Nguyen, M.C.; Do, T.T.; Soejarto, D.D.; et al. Antimalarial compounds from Rhaphidophora decursiva. J. Nat. Prod. 2001, 64, 772-777. [CrossRef] [PubMed]

23. Zhang, H.J.; Qiu, S.; Tamez, P.; Tan, G.T.; Aydogmus, Z.; Nguyen, V.H.; Nguyen, M.C.; Angerhofer, C.; Soejarto, D.D.; Pezzuto, J.M.; et al. Antimalarial agents from plants II. Decursivine, a new antimalarial indole alkaloid from Rhaphidophora decursiva. Pharm. Biol. 2002, 40, 221-224. [CrossRef]

24. Zhang, H.J.; Tamez, P.A.; Aydoqmus, Z.; Tan, G.T.; Saikawa, Y.; Hashimoto, K.; Nakata, M.; Hung, N.V.; Xuan, L.T.; Cuong, N.M.; et al. Antimalarial agents from plants. III. Trichothecenes from Ficus fistulosa and Rhaphidophora decursiva. Planta Med. 2002, 68, 1088-1091. [CrossRef] [PubMed]

25. Libman, A.; Zhang, H.; Ma, C.; Southavong, B.; Sydara, K.; Bouamanivong, S.; Tan, G.T.; Fong, H.H.; Soejarto, D.D. A first new antimalarial pregnane glycoside from Gongronema napalense. Asian J. Tradit. Med. 2008, 3, 203-210. [PubMed]

26. He, Z.D.; Ma, C.Y.; Tan, G.T.; Sydara, K.; Tamez, P.; Southavong, B.; Bouamanivong, S.; Soejarto, D.D.; Pezzuto, J.M.; Fong, H.H.; et al. Rourinoside and rouremin, antimalarial constituents from Rourea minor. Phytochemistry 2006, 67, 1378-1384. [CrossRef] [PubMed]

27. Ma, C.Y.; Musoke, S.F.; Tan, G.T.; Sydara, K.; Bouamanivong, S.; Southavong, B.; Soejarto, D.D.; Fong, H.H.; Zhang, H.J. Study of antimalarial activity of chemical constituents from Diospyros quaesita. Chem. Biodivers. 2008, 5, 2442-2448. [CrossRef] [PubMed]

28. He, Z.D.; Ma, C.Y.; Zhang, H.J.; Tan, G.T.; Tamez, P.; Sydara, K.; Bouamanivong, S.; Southavong, B.; Soejarto, D.D.; Pezzuto, J.M.; et al. Antimalarial constituents from Nauclea orientalis (L.) L. Chem. Biodivers. 2005, 2, 1378-1386. [CrossRef] [PubMed]

29. Ma, C.; Zhang, H.J.; Tan, G.T.; Hung, N.V.; Cuong, N.M.; Soejarto, D.D.; Fong, H.H. Antimalarial compounds from Grewia bilamellata. J. Nat. Prod. 2006, 69, 346-350. [CrossRef] [PubMed]

30. Schwikkard, S.; van Heerden, F.R. Antimalarial activity of plant metabolites. Nat. Prod. Rep. 2002, 19, 675-692. [CrossRef] [PubMed]

31. Prawat, U.; Phupornprasert, D.; Butsuri, A.; Salae, A.W.; Boonsri, S.; Tuntiwachwuttikul, P. Flavonoids from Friesodielsia discolor. Phytochem. Lett. 2012, 5, 809-813. [CrossRef]

32. Mueller, D.; Davis, R.A.; Duffy, S.; Avery, V.M.; Camp, D.; Quinn, R.J. Antimalarial activity of azafluorenone alkaloids from the Australian tree Mitrephora diversifolia. J. Nat. Prod. 2009, 72, 1538-1540. [CrossRef] [PubMed]

33. Promchai, T.; Jaidee, A.; Cheenpracha, S.; Trisuwan, K.; Rattanajak, R.; Kamchonwongpaisan, S.; Laphookhieo, S.; Pyne, S.G.; Ritthiwigrom, T. Antimalarial Oxoprotoberberine Alkaloids from the Leaves of Miliusa cuneata. J. Nat. Prod. 2016, 79, 978-983. [CrossRef] [PubMed]

34. Vitalini, S.; Beretta, G.; Iriti, M.; Orsenigo, S.; Basilico, N.; Dall'Acqua, S.; Iorizzi, M.; Fico, G. Phenolic compounds from Achillea millefolium L. and their bioactivity. Acta Biochim. Pol. 2011, 58, 203-209. [PubMed]

35. Chung, I.M.; Seo, S.H.; Kang, E.Y.; Park, W.H.; Park, S.D.; Moon, H.I. Antiplasmodial activity of isolated compounds from Carpesium divaricatum. Phytother. Res. 2010, 24, 451-453. [CrossRef] [PubMed]

36. Köhlera, I.; Jenett-Siems, K.; Kraft, C.; Siems, K.; Abbiw, D.; Bienzle, U.; Eich, E. Herbal remedies traditionally used against malaria in Ghana: Bioassay-guided fractionation of Microglossa pyrifolia (Asteraceae). Z. Naturforsch. C 2002, 57, 1022-1027. [CrossRef] 
37. Bitew, H.; Mammo, W.; Hymete, A.; Yeshak, M.Y. Antimalatial activity of acetylenic thiophenes from Echinops hoehnelii Schweinf. Molecules 2017, 22, 1965. [CrossRef] [PubMed]

38. Cai, S.; Risinger, A.L.; Nair, S.; Peng, J.; Anderson, T.J.; Du, L.; Powell, D.R.; Mooberry, S.L.; Chichewicz, R.H. Identification of compounds with efficacy against malaria parasites from common North American plants. J. Nat. Prod. 2015, 79, 490-498. [CrossRef] [PubMed]

39. Uchôa, V.T.; de Paula, R.C.; Krettli, L.G.; Santan, A.E.G.; Krettli, A.U. Antimalarial activity of compounds and mixed fractions of Cecropia pachystachya. Drug Dev. Res. 2010, 71, 82-91. [CrossRef]

40. Zhou, B.; Wu, Y.; Dalal, S.; Merino, E.F.; Liu, Q.F.; Xu, C.H.; Tao, Y.; Ding, J.; Kingston, D.G.I.; Cassera, M.B.; et al. Nanomolar antimalarial agents against chloroquine-resistant Plasmodium falciparum from medicinal plants and their structure-activity relationships. J. Nat. Prod. 2017, 80, 96-107. [CrossRef] [PubMed]

41. Uys, A.C.; Malan, S.F.; van Dyk, S.; van Zyl, R.L. Antimalarial compounds from Parinari capensis. Bioorg. Med. Chem. Lett. 2002, 12, 2167-2169. [CrossRef]

42. Auranwiwat, C.; Laphookhieo, S.; Rattanajak, R.; Kamchonwongpaisan, S.; Pyne, S.G.; Ritthiwigrom, T. Antimalarial polyoxygenated and prenylated xanthones from the leaves and branches of Garcinia mckeaniana. Tetrahedron 2016, 72, 6837-6842. [CrossRef]

43. Graziose, R.; Rojas-Silva, P.; Rathinasabapathy, T.; Dekoc, C.; Grace, M.H.; Poulev, A.; Ann, L.M.; Smith, P.; Raskin, I. Antiparasitic compounds from Cornus florida L. with activities against Plasmodium falciparum and Leishmania tarentolae. J. Ethnopharmacol. 2012, 142, 456-461. [CrossRef] [PubMed]

44. Banzouzi, J.T.; Soh, P.N.; Mbatchi, B.; Cavé, A.; Ramos, S.; Retailleau, P.; Rakotonandrasana, O.; Berry, A.; Benoit-Vical, F. Cogniauxia podolaena: Bioassay-guided fractionation of defoliated stems, isolation of active compounds, antiplasmodial activity and cytotoxicity. Planta Med. 2008, 74, 1453-1456. [CrossRef] [PubMed]

45. Hadi, V.; Hotard, M.; Ling, T.; Salinas, Y.G.; Palacios, G.; Connelly, M.; Rivas, F. Evaluation of Jatropha isabelli natural products and their synthetic analogs as potential antimalarial therapeutic agents. Eur. J. Med. Chem. 2013, 65, 376-380. [CrossRef] [PubMed]

46. Seephonkai, P.; Sangdee, A.; Bunchalee, P.; Pyne, S.G. Cytotoxic and antiplasmodial compounds from the roots of Strophioblachia fimbricalyx. J. Nat. Prod. 2009, 72, 1892-1894. [CrossRef] [PubMed]

47. Ajaiyeoba, E.O.; Ogbole, O.O.; Abiodun, O.O.; Ashidi, J.S.; Houghton, P.J.; Wright, C.W. Cajachalcone: An Antimalarial Compound from Cajanus cajan Leaf Extract. J. Parasitol. Res. 2013, 2013, 703781. [CrossRef] [PubMed]

48. Ramanandraibe, V.; Grellier, P.; Martin, M.T.; Deville, A.; Joyeau, R.; Ramanitrahasimbola, D.; Mouray, E.; Rasoanaivo, P.; Mambu, L. Antiplasmodial phenolic compounds from Piptadenia pervillei. Planta Med. 2008, 74, 417-421. [CrossRef] [PubMed]

49. Samoylenko, V.; Ashfaq, M.K.; Jacob, M.R.; Tekwani, B.L.; Khan, S.I.; Manly, S.P.; Joshi, V.C.; Walker, L.A.; Muhammad, I. Indolizidine, antiinfective and antiparasitic compounds from Prosopis glandulosa var. Glandulosa. J. Nat. Prod. 2009, 72, 92-98. [CrossRef] [PubMed]

50. Mbwambo, Z.H.; Apera, S.; Moshi, M.J.; Kapingu, M.C.; Van Miert, S.; Claeys, M.; Brun, R.; Cos, P.; Pieters, L.; Vlietinck, A. Anthranoid compounds with antiprotozoal activity from Vismia orientalis. Planta Med. 2004, 70, 706-710. [CrossRef] [PubMed]

51. Ndjakou Lenta, B.; Devkota, K.P.; Ngouela, S.; Fekam Boyom, F.; Naz, Q.; Choudhary, M.I.; Tsamo, E.; Rosenthal, P.J.; Sewald, N. Anti-plasmodial and cholinesterase inhibiting activities of some constituents of Psorospermum glaberrimum. Chem. Pharm. Bull. 2008, 56, 222-226. [CrossRef] [PubMed]

52. Zhu, S. Small Molecules with Antimalarial Activity. U.S. Patent 2013/0023552 A1, 24 January 2013.

53. Kirmizibekmez, H.; Calis, I.; Perozzo, R.; Brun, R.; Dönmez, A.A.; Linden, A.; Rüedi, P.; Tasdemir, D. Inhibiting activities of the secondary metabolites of Phlomis brunneogaleata against parasitic protozoa and plasmodial enoyl-ACP Reductase, a crucial enzyme in fatty acid biosynthesis. Planta Med. 2004, 70, 711-717. [CrossRef] [PubMed]

54. Kamatou, G.P.P.; Van Zyl, R.L.; Davids, H.; Van Heerden, F.R.; Lourens, A.C.U.; Viljoen, A.M. Antimalarial and anticancer activities of selected South African Salviaspecies and isolated compounds from S. radula. S. Afr. J. Bot. 2008, 74, 238-243. [CrossRef]

55. Tchinda, A.T.; Tamze, V.; Ngono, A.R.N.; Ayimele, G.A.; Cao, M.; Angenot, L.; Frédérich, M. Alkaloids from the stem bark of Strychnos icaja. Phytochem. Lett. 2012, 5, 108-113. [CrossRef] 
56. Upadhyaya, H.C.; Sisodia, B.S.; Agrawal, J.; Pal, A.; Darokar, M.P.; Srivastava, S.K. Antimalarial potential of extracts and isolated compounds from four species of genus Ammannia. Med. Chem. Res. 2014, 23, 870-876. [CrossRef]

57. Sprogøe, K.; Staek, D.; Ziegler, H.L.; Jensen, T.H.; Holm-Møller, S.B.; Jaroszewski, J.W. Combining HPLC-PDA-MS-SPE-NMR with circular dichroism for complete natural product characterization in crude extracts: Levorotatory gossypol in Thespesia danis. J. Nat. Prod. 2008, 71, 516-519. [CrossRef] [PubMed]

58. Buchanan, M.S.; Davis, R.A.; Duffy, S.; Avery, V.M.; Quinn, R.J. Antimalarial benzylisoquinoline alkaloid from the rainforest tree Doryphora sassafras. J. Nat. Prod. 2009, 72, 1541-1543. [CrossRef] [PubMed]

59. Mbah, J.A.; Tane, P.; Ngadjui, B.T.; Connolly, J.D.; Okunji, C.C.; Iwu, M.M.; Schuster, B.M. Antiplasmodial agents from the leaves of Glossocalyx brevipes. Planta Med. 2004, 70, 437-440. [PubMed]

60. Kubo, M.; Yatsuzuka, W.; Matsushima, S.; Harada, K.; Inoue, Y.; Miyamoto, H.; Matsumoto, M.; Fukuyama, Y. Antimalarial phenanthroindolizine alkaloids from Ficus septica. Chem. Pharm. Bull. 2016, 64, 957-960. [CrossRef] [PubMed]

61. Rangkaew, N.; Suttisri, R.; Moriyasu, M.; Kawanishi, K. A new acyclic diterpene acid and bioactive compounds from Knema glauca. Arch. Pharm. Res. 2009, 32, 685-692. [CrossRef] [PubMed]

62. Rukachaisirikul, T.; Siriwattanakit, P.; Sukcharoenphol, K.; Wongvein, C.; Ruttanaweang, P.; Wongwattanavuch, P.; Suksamrarn, A. Chemical constituents and bioactivity of Piper sarmentosum. J. Ethnopharmacol. 2004, 93, 173-176. [CrossRef] [PubMed]

63. Sáez Vega, A.; Rojanoa, B.; Blair, S.; Segura, C.; Figadere, B.; Seone, B.; Grellierf, P.; Sáeza, J. Antimalarials and antioxidants compounds from Piper tricuspe (Piperaceae). Pharmacologyonline 2008, 1, 1-8.

64. Lacroix, D.; Prado, S.; Kamoga, D.; Kasenene, J.; Bodo, B. Structure and in vitro antiparasitic activity of constituents of Citropsis articulata root bark. J. Nat. Prod. 2011, 74, 2286-2289. [CrossRef] [PubMed]

65. Cebrián-Torrejón, G.; Spelman, K.; Leblanc, K.; Muñoz-Durango, K.; Gutiérrez, S.T.; Ferreira, M.E.; de Arias, A.R.; Figadere, B.; Fournet, A.; Maciuk, A.; et al. The antiplasmodium effects of a traditional South American remedy: Zanthoxylum chiloperone var. angustifolium against chloroquine resistant and chloroquine sensitive strains of Plasmodium falciparum. Rev. Bras. Farmacogn. Braz. J. Pharmacogn. 2011, 21, 652-661. [CrossRef]

66. Kuo, P.C.; Damu, A.G.; Lee, K.H.; Wu, T.S. Cytotoxic and antimalarial constituents from the roots of Eurycoma longifolia. Bioorg. Med. Chem. 2004, 12, 537-544. [CrossRef] [PubMed]

67. Tada, H.; Yasuda, F.; Otani, K.; Doteuchi, M.; Ishihara, Y.; Shiro, M. New antiulcer quassinoids from Eurycoma longifolia. Eur. J. Med. Chem. 1991, 26, 345-349. [CrossRef]

68. De Andrade-Neto, V.F.; Pohlit, A.M.; Pinto, A.C.; Silva, E.C.; Nogueir, K.L.; Melo, M.R.; Henrique, M.C.; Amorim, R.C.; Silva, L.F.; Costa, M.R.; et al. In vitro inhibition of Plasmodium falciparum by substances isolated from Amazonian antimalarial plants. Mem. Inst. Oswaldo Cruz 2007, 102, 359-365. [CrossRef] [PubMed]

69. Tegar, M.; Purnomo, H. Tea leaves extracted as anti-malaria based on molecular docking plants. Procedia Environ. Sci. 2013, 17, 188-194. [CrossRef]

70. Ludere, M.T.; van Ree, T.; Vleggaa, R. Isolation and relative stereochemistry of lippialactone, a new antimalarial compound from Lippia javanica. Fitoterapia 2013, 86, 188-192. [CrossRef] [PubMed]

71. Karunamoorthi, K.; Tsehaye, E. Ethnomedicinal knowledge, belief and self-reported practice of local inhabitants on traditional antimalarial plants and phytotherapy. J. Ethnopharmacol. 2012, 141, 143-150. [CrossRef] [PubMed]

72. Adepiti, A.O.; Elujoba, A.A.; Bolaji, O.O. In vivo antimalarial evaluation of MAMA decoction on Plasmodium berghei in mice. Parasitol. Res. 2014, 113, 505-511. [CrossRef] [PubMed]

73. Ajaiyeoba, E.O.; Abiodun, O.O.; Falade, M.O.; Ogbole, N.O.; Ashidi, J.S.; Happi, C.T.; Akinboye, D.O. In vitro cytotoxicity studies of 20 plants used in Nigerian antimalarial ethnomedicine. Phytomedicine 2006, 13, 295-298. [CrossRef] [PubMed]

74. Ogunkunle, A.T.; Oyelakin, T.M.; Enitan, A.O.; Oyewole, F.E. A quantitative documentation of the composition of two powdered herbal formulations (antimalarial and haematinic) using ethnomedicinal information from ogbomoso, Nigeria. Evid. Based Complement. Altern. Med. 2014, 2014, 1-8. [CrossRef] [PubMed]

75. Rasoanaivo, P.; Petitjean, A.; Ratsimamanga-Urverg, S.; Rakoto-Ratsimamanga, A. Medicinal plants used to treat malaria in Madagascar. J. Ethnopharmacol. 1992, 37, 117-127. [CrossRef] 
76. Ojewole, J.A.; Mawoza, T.; Chiwororo, W.D.; Owira, P.M. Sclerocarya birrea (A. Rich) Hochst. ['Marula'] (Anacardiaceae): A review of its phytochemistry, pharmacology and toxicology and its ethnomedicinal uses. Phytother. Res. 2010, 24, 633-639. [PubMed]

77. Qinghaosu Antimalaria Coordinating Research Group. Antimalaria studies on Qinghaosu. Chin. Med. J. 1979, 92, 811-816.

78. Kitua, A.Y.; Malebo, H.M. Malaria control in Africa and the role of traditional medicine. In Traditional Medicinal Plants and Malaria, 1st ed.; Willcox, M., Bodeker, G., Rasoanaivo, P., Addae-Kyereme, J., Eds.; CRC Press: Boca Raton, FL, USA, 2004; pp. 2-20.

79. Zeleke, G.; Kebebe, D.; Mulisa, E.; Gashe, F. In vivo antimalarial activity of the solvent fractions of fruit and root of Carica papaya Linn (Caricaceae) against Plasmodium berghei in Mice. Evid. Based Complement. Altern. Med. 2017, 2017, 3121050. [CrossRef] [PubMed]

80. Muregi, F.W.; Ishih, A.; Miyase, T.; Suzuki, T.; Kino, H.; Amano, T.; Mkoji, G.M.; Terada, M. Antimalarial activity of methanolic extracts from plants used in Kenyan ethnomedicine and their interactions with chloroquine (CQ) against a CQ-tolerant rodent parasite, in mice. J. Ethnopharmacol. 2007, 111, 190-195. [CrossRef] [PubMed]

81. Abiodun, O.O.; Gbotosho, G.O.; Ajaiyeoba, E.O.; Happi, C.T.; Falade, M.; Wittlin, S.; Sowunmi, A.; Brun, R.; Oduola, A. In vitro antiplasmodial activity and toxicity assessment of some plants from Nigerian ethnomedicine. Pharm. Biol. 2011, 49, 9-14. [CrossRef] [PubMed]

82. Abiodun, O.O.; Gbotosho, G.O.; Ajaiyeoba, E.O.; Happi, C.T.; Hofer, S.; Wittlin, S.; Sowunmi, A.; Brun, R.; Oduola, A.M. Comparison of SYBR Green I-, PicoGreen-, and $\left[{ }^{3} \mathrm{H}\right]$-hypoxanthine-based assays for in vitro antimalarial screening of plants from Nigerian ethnomedicine. Parasitol. Res. 2010, 106, 933-939. [CrossRef] [PubMed]

83. Patel, J.R.; Tripathi, P.; Sharma, V.; Chauhan, N.S.; Dixit, V.K. Phyllanthus amarus: Ethnomedicinal uses, phytochemistry and pharmacology: A review. J. Ethnopharmacol. 2011, 138, 286-313. [CrossRef] [PubMed]

84. Adjobimey, T.; Edaye, I.; Lagnika, L.; Gbenou, J.; Moudachirou, M.; Sanni, A. Activités antiplasmodiales in vitro de quelques plantes antipaludiques de la pharmacopée béninoise. Comptes Rendus Chim. 2004, 7, 1023-1027. [CrossRef]

85. Upadhyay, B.; Parveen Dhaker, A.K.; Kumar, A. Ethnomedicinal and ethnopharmaco-statistical studies of Eastern Rajasthan. Indian J. Ethnopharmacol. 2010, 129, 64-86. [CrossRef] [PubMed]

86. Sadiq, M.B.; Tharaphan, P.; Chotivanich, K.; Tarning, J.; Anal, A.K. In vitro antioxidant and antimalarial activities of leaves, pods and bark extracts of Acacia nilotica (L.) Del. BMC Complement. Altern. Med. 2017, 17, 372. [CrossRef] [PubMed]

87. Zhu, S.; Zhang, Q.; Gudise, C.; Wei, L.; Smith, E.; Zeng, Y. Synthesis and biological evaluation of febrifugine analogues as potential antimalarial agents. Bioorg. Med. Chem. 2009, 17, 4496-4502. [CrossRef] [PubMed]

88. Satish, P.V.V.; Sunita, K. Antimalarial efficacy of Pongamia pinnata (L) Pierre against Plasmodium falciparum (3D7 strain) and Plasmodium berghei (ANKA). BMC Complement. Altern. Med. 2017, 17, 458. [CrossRef] [PubMed]

89. Moghadamtousi, S.Z.; Goh, B.H.; Chan, C.K.; Shabab, T.; Kadir, H.A. Biological activities and phytochemicals of Swietenia macrophylla King. Molecules 2013, 18, 10465-10483. [CrossRef] [PubMed]

90. Falade, M.O.; Akinboye, D.O.; Gbotosho, G.O.; Ajaiyeob, E.O.; Happi, T.C.; Abiodun, O.O.; Oduola, A.M. In Vitro and In Vivo Antimalarial Activity of Ficus thonningii Blume (Moraceae) and Lophira alata Banks (Ochnaceae), Identified from the Ethnomedicine of the Nigerian Middle Belt. J. Parasitol. Res. 2014, 2014, 1-6. [CrossRef] [PubMed]

91. Teinkela, J.E.M.; Noundou, X.S.; Nguemfo, E.L.; Meyer, F.; Wintjens, R.; Isaacs, M.; Mpondo Mpondo, A.E.; Hoppe, H.C.; Krause, R.W.M.; Azebaze, A.G.B. Biological activities of plant extracts from Ficus elastica and Selaginella vogelli: An antimalarial, antitrypanosomal and cytotoxity evaluation. Saudi J. Biol. Sci. 2018, 25, 117-122. [CrossRef] [PubMed]

92. Ansah, C.; Gooderham, N.J. The popular herbal antimalarial, extract of Cryptolepis sanguinolenta, is potently cytotoxic. Toxicol. Sci. 2002, 70, 245-251. [CrossRef] [PubMed]

93. Kazuki, T.; Yasunori, Y.; Masao, K. Constituents of the leaves and roots of Ligularia stenocephala Matsum. J. Nat. Med. 2006, 60, 329-330. 
94. Lane, A.L.; Stout, E.P.; Lin, A.S.; Prudhomme, J.; le Roch, K.; Fairchild, C.R.; Franzblau, S.G.; Hay, M.E.; Aalbersberg, W.; Kubanek, J. Antimalarial bromophycolides J-Q from the Fijian red alga Callophycus serratus. J. Org. Chem. 2009, 74, 2736-2742. [CrossRef] [PubMed]

95. Teasdale, M.E.; Prudhomme, J.; Torres, M.; Braley, M.; Cervantes, S.; Bhatia, S.C.; la Clair, J.J.; le Roch, K.; Kubanek, J. Pharmacokinetics, metabolism, and in vivo efficacy of the antimalarial natural product bromophycolide A. ACS Med. Chem. Lett. 2013, 4, 989-993. [CrossRef] [PubMed]

96. Yang, F.; Wang, R.P.; Xu, B.; Yu, H.B.; Ma, G.Y.; Wang, G.F.; Dai, S.W.; Zhang, W.; Jiao, W.H.; Song, S.J.; et al. New antimalarial norterpene cyclic peroxides from Xisha Islands sponge Diacarnus megaspinorhabdosa. Bioorg. Med. Chem. Lett. 2016, 526, 2084-2087. [CrossRef] [PubMed]

97. World Health Organization. World Health Report 2002; WHO Press: Geneva, Switzerland, 2002.

98. De Ridder, S.; van der Kooy, F.; Robert Verpoorte, R. Artemisia annua as a self-reliant treatment for malaria in developing countries. J. Ethnopharmacol. 2008, 120, 302-314. [CrossRef] [PubMed]

99. Dharani, N.; Rukunga, G.; Abiy Yenesew, A.; Mbora, A.; Mwaura, L.; Dawson, I.; Jamnadass, R. Common Antimalarial Trees and Shrubs of East Africa; World Agroforestry Centre and the Kenya Medical Research Institute: Nairobo, Kenya, 2010.

100. Loua, J.; Traore, M.S.; Camara, A.; Balde, M.A.; Maes, L.; Pieters, L.; Balde, A.M. Biological and phytochemical investigations on Caesalpinia benthamiana, a plant traditionally used as antimalarial in Guinea. Evid. Based Complement. Altern. Med. 2017, 2017, 9438607. [CrossRef] [PubMed]

(C) 2018 by the authors. Licensee MDPI, Basel, Switzerland. This article is an open access article distributed under the terms and conditions of the Creative Commons Attribution (CC BY) license (http://creativecommons.org/licenses/by/4.0/). 